\title{
Extended scalar sectors, effective operators and observed data
}

\author{
Atri Dey, Jayita Lahiri and Biswarup Mukhopadhyaya \\ Regional Centre for Accelerator-based Particle Physics, \\ Harish-Chandra Research Institute, HBNI, \\ Chhatnag Road, Jhunsi, Allahabad - 211 019, India \\ E-mail: atridey@hri.res.in, jayitalahiri@hri.res.in, \\ biswarup@hri.res.in
}

ABSTRACT: The available data on the $125 \mathrm{GeV}$ scalar $h$ is analysed to explore the room for new physics in the electroweak symmetry breaking sector. The first part of the study is model-independent, with $h$ couplings to standard model particles scaled by quantities that are taken to be free parameters. At the same time, the additional loop contributions to $h \rightarrow \gamma \gamma$ and $h \rightarrow Z \gamma$, mediated by charged scalar contributions in the extended scalar sector, are treated in terms of gauge-invariant effective operators. Having justified this approach for cases where the concerned scalar masses are a little above the $Z$-boson mass, we fit the existing data to obtain marginalized $1 \sigma$ and $2 \sigma$ regions in the space of the coefficients of such effective operators, where the limit on the $h \rightarrow Z \gamma$ branching ratio is used as a constraint. The correlation between, say, the gluon fusion and vector-boson fusion channels, as reflected in a non-diagonal covariance matrix, is taken into account. After thus obtaining model-independent fits, the allowed values of the coefficients are translated into permissible regions of the parameter spaces of several specific models. In this spirit we constrain four different types of two Higgs doublet models, and also models with one or two $Y=2$ scalar triplets, taking into account the correlatedness of the scale factors in $h$-interactions and the various couplings of charged Higgs states in each extended scenario.

Keywords: Beyond Standard Model, Higgs physics, Electroweak interaction, Branching fraction, Hadron-Hadron scattering (experiments)

ARXIV EPRINT: 1808.04869 


\section{Contents}

1 Introduction 1

2 Modified Higgs couplings $\quad 4$

$\begin{array}{lll}3 & \text { The global fit } & 7\end{array}$

4 Extended Higgs models and dimension-6 operators 11

4.1 Two Higgs doublet models 14

$\begin{array}{lll}\text { 4.1.1 Type-I 2HDM } & 15\end{array}$

$\begin{array}{lll}4.1 .2 & \text { Type-II 2HDM } & 17\end{array}$

$\begin{array}{lll}\text { 4.1.3 Lepton-specific 2HDM } & 18\end{array}$

4.1.4 Flipped 2HDM 20

4.1.5 Comparison among various 2HDMs 21

4.2 Higgs triplet models 24

4.2.1 A single-triplet model 25

$\begin{array}{lll}4.2 .2 & \text { A model with two triplets } & 27\end{array}$

5 Conclusion 28

$\begin{array}{lr}\text { A Decay amplitudes in 2HDM } & 29\end{array}$

B Decay amplitudes in models with triplet scalar(s) $\quad 31$

B.1 Single triplet case 31

B.2 Two-triplet case 32

\section{Introduction}

The ATLAS and CMS experiments at the Large Hadron Collider (LHC) [1] have discovered a new boson mass $125 \mathrm{GeV}$. Though the properties of this particle are similar to those of the Higgs boson predicted in the standard model (SM) of electroweak interactions, everyone is on the look-out for any small difference that may reveal the participation of some new physics. It is thus imperative to closely examine all interactions (including supposedly 'effective' ones) of this particle with SM fermions and gauge bosons. The accumulation of data has helped a lot in pinning down uncertainties here; scopes of departure, however, still remain. Besides, issues ranging from naturalness of the Higgs mass to the dark matter content of our universe continue to provide impetus for physics beyond the standard model (BSM). Theoretical models extending the SM, including those augmenting the electroweak symmetry breaking sector, are thus explored. It is a natural endeavour to link the contributions of such new physics, in the form of modified Higgs interaction strengths as well as effective operators generated by them, to the departure from unity in the Higgs signal strengths in various final states $f$, defined as $\mu^{f}=\frac{\sigma^{f}}{\sigma_{\mathrm{SM}}^{f}}$. Fitting the available data with 
various $\mu^{f}$, therefore, enables one to analyze allowed strengths of effective operators generated in various models. These ultimately constrain the model parameters themselves. This can be helpful since new particles belonging to some new physics scenario often have low production rates in accelerators. They instead make their presence felt (i) by participating off-shell, and leading to higher-dimensional effective operators, and (ii) by multiplicatively modifying the coupling strength(s) of the $125 \mathrm{GeV}$ scalar to the other SM particles. The present study is devoted to such a situation.

Interestingly, significant constraints arise from the signal strengths for the loop induced decay channel such as $h \rightarrow \gamma \gamma$. $h \rightarrow Z \gamma$, although yet unobserved, can also provide strong limits on new physics contributions, especially with accumulating luminosity. These limits can be coupled with those arising from tree-level decay modes such as $h \rightarrow W W, Z Z$ etc. where perceptible effects can come mostly via scaling of the SM coupling by a factor $\kappa$.

The effect of high scale physics on low-energy processes can be formulated in terms of higher dimension operators in the Lagrangian, which will be suppressed by the new physics scale $\Lambda$. These higher-dimensional operators can be derived from an $\mathrm{SU}(2)_{L} \times \mathrm{U}(1)_{Y^{-}}$ invariant basis, as they result from physics corresponding to a scale much higher than the electroweak scale. It is not possible to construct dimension-5 operators of this kind. Therefore we concentrate on dimension- 6 gauge-invariant operators. As mentioned above, we mainly focus on two loop-induced decays, namely, $h \rightarrow \gamma \gamma$ and $h \rightarrow Z \gamma$. Thus the most general dimension-6 operators which give rise to $h V V$ vertices $(V=W, Z)$ get constrained from the rates of these processes, as tree-level processes are unlikely to bear clear enough stamps of such operators. Constraints on these higher dimensional operators as well as the scale factors $\kappa$ have been extensively studied on the basis of electroweak precision tests and global fits of Higgs data in [2-16]. However such studies contain no attempt to link the analysis to specific theoretical models, especially those involving extensions of the Higgs sector. Linking them will need to take cognizance of the correlation between the $\kappa$-factors and the Wilson coefficients of the higher-dimensional operators, via the parameters of the model(s) corcerned. Such correlated linking is the quintessence of the present paper.

We have adopted a slightly different formulation of new physics contributions to the aforementioned loop-induced decays. The consequences of new physics have been divided into two categories. The first of these is the scaling of the couplings $h t \bar{t}, h b \bar{b}, h \tau \bar{\tau}, h V V$, where modifications to the SM couplings are inevitable when additional fields mixing with the ones in SM are present. As we have already mentioned, such modifications usually override the effects of higher-dimensional operators. Such scaling also affects loop effects such as $h \rightarrow \gamma \gamma, Z \gamma$, via modified vertices in the loop where, W's or top quarks are involved, and the corresponding amplitudes can be written down in terms of the scale factors $\kappa$. The second category consists in loop diagrams mediated by new particles such as charged scalars. Their contributions to loop amplitudes, we argue, can be expressed in isolation in terms of effective couplings. As shown in section 4, these couplings, at least those ensuing from additional scalar fields, can be treated as ones derived by the aforesaid gauge-invariant dimension- 6 operators, so long as the masses of the new particles are gauge-invariant and at least a little above the electroweak symmetry breaking (EWSB) scale. 
Keeping this in mind, we perform a global fit of the currently available Higgs data to constrain the full parameter space including scale factors and the Wilson coefficients corresponding to various dimension-6 operators. While using the Higgs signal strength data in various channels we take into account the correlation between various production processes such as gluon fusion and vector-boson fusion, thus including non-diagonal covariant matrices in our analysis. Model-independent $2 \sigma$ regions for the $\kappa$ 's well as the Wilson coefficients are thus obtained.

We select specific models in the next step of the analysis. The present study is restricted to additional scalars, and includes various kinds of two-Higgs doublet models (2HDM) as well as those with one and two scalar triplets of the kind introduced in Type-II seesaw mechanism. The marginalized $2 \sigma$ regions in the space of dimension- 6 operators are then recast, keeping track of the correlation between the scale factors $\kappa$ and the Wilson coefficients for each model. These are finally translated into constraints in the space of masses and coupling strengths pertaining to all the models.

There is as of now just an upper limit on the signal strength in the $Z \gamma$ channel. Keeping this in mind, We further construct a ratio involving the signal strengths for $Z \gamma$ and $\gamma \gamma$, which can help us distinguish between various new physics models. If a charged scalar (which can in principle contribute to both the above final states) is discovered later, this ratio will help us in narrowing down possibilities regarding the $\mathrm{SU}(2)$ multiplet it could be a part of. In case of a future discovery of one or more heavy Higgs, this can enable one to distinguish among various theories with extended electroweak symmetry breaking sectors, following the strategy developed in this paper.

In a nutshell, the novel features in our study are the following:

- We have segregated two types of modification, namely, those in tree-level couplings of the Higgs to SM particles, and the contribution of loop integrals involving BSM mediators to processes disallowed at the tree-level.

- The latter contribution mentioned above has been parametrized in isolation in terms of dimension- 6 operators, and the legitimacy of doing this has been demonstrated.

- The effect of the former modification to tree-level as well as loop-induced decays have been taken into account, while the contribution of dimension- 6 operators have been assumed, as a pragmatic measure, to be confined to loop-induced processes.

- The effective operators has been shown to work for just a subset of the one-loop diagrams contributing to effective $h \gamma \gamma$ and $h Z \gamma$ couplings, even when the masses of new scalars running in these loops are not much above the electroweak scale.

- Non-diagonal covariance matrices have been used.

- We then establish a connection between allowed parameter regions in the modelindependent approach with those of specific new physics models. 
- Finally we propose using the ratio of rates in the $\gamma \gamma$ and $Z \gamma$ channels, potentially observable at the LHC with accumulating luminosity. This allows disentanglement of various types of new physics.

The paper is organized as follows. In section 2, we discuss the parametrization of modified Higgs couplings involving both scaling and higher-dimensional operators. A global fit carried out on an 8-dimensional parameter space, taking into account the correlation among various production channels of the Higgs particle, is reported in section 3. Section 4 contains a discussion of several extended Higgs models, wherefrom we arrive at the resulting constraints on the corresponding models. We summarize and conclude in section 5 . Some expressions relevant to the loop amplitudes in $h \rightarrow \gamma \gamma$ and $h \rightarrow Z \gamma$ are listed in the appendices.

\section{Modified Higgs couplings}

As mentioned in the previous section, there can be an extended Higgs sector comprising of additional neutral and charged scalars. Their mixing may cause the coupling of the $125 \mathrm{GeV}$ scalar to SM particles to be modified. ${ }^{1}$ Modification may generally occur in two ways. First, there can be scaling of the Higgs couplings, with unaltered Lorentz structure of the corresponding vertices. Such scaling can be expressed as

$$
\begin{aligned}
\tilde{g}_{h V V} & =\kappa_{v} \times g_{h V V} \\
\tilde{g}_{h t \bar{t}} & =\kappa_{t} \times g_{h t \bar{t}} \\
\tilde{g}_{h b \bar{b}} & =\kappa_{b} \times g_{h b \bar{b}} \\
\tilde{g}_{h \tau \bar{\tau}} & =\kappa_{\tau} \times g_{h \tau \bar{\tau}}
\end{aligned}
$$

Where $g_{h V V}, g_{h t \bar{t}}, g_{h b \bar{b}}$ and $g_{h \tau \bar{\tau}}$ are the couplings of the Higgs to the gauge bosons and the fermions in the SM. The couplings of Higgs to $W$ boson and $Z$ boson are scaled in the same way here. This is because the custodial SU(2) symmetry, ensuring tree-level unity of the $\rho$-parameter, is otherwise at stake, unless additional contributions to $\rho$ are built into the theory.

Moreover, there can be heavy states running in the loop modifying Higgs couplings. A general approach to parametrize such modification is to express it in terms of gaugeinvariant higher-dimensional effective operators. Here one normally expects that the new physics here is at a high scale (at least a $\mathrm{TeV}$ or thereabout), thus justifying $\mathrm{SU}(2)_{L} \times$ $\mathrm{U}(1)_{Y}$ invariance of the operator(s) involved. However, one can see that such TeV-scale suppressants may arise even for lower masses in the loop, thanks to the factors $\mathcal{O} \sim 16 \pi^{2}$ in the loop integrals. Gauge invariance of the Wilson coefficients, will, require that the masses of particles running in the loops should arise from $\mathrm{SU}(2)_{L} \times \mathrm{U}(1)_{Y}$ invariant terms. This requirement is satisfied in each model, involving extension of the electroweak symmetry breaking sector, used by us for illustration.

\footnotetext{
${ }^{1}$ In practice, similar modifications may arise due to the presence of additional fermions and gauge bosons, having mixing with the SM particles. We specifically mention additional scalars because the modeldependent part of our analysis is confined to such scenarios only.
} 
All Higgs interactions should in principle be modified via such operators. However, couplings which exist at the SM at tree-level, namely, $h W W, h Z Z, h t \bar{t}, h b \bar{b}, h \tau \bar{\tau}$, are rather nominally affected by higher-dimensional terms with (at least) TeV-scale suppression. Thus one can, for all practical purpose, neglect such modification compared to the tree-level couplings and the scaling effects, if any. On the other hand, the $h \gamma \gamma$ and $h Z \gamma$ vertices which appear only at the one-loop level [17] are expected to have relatively non-negligible contribution from the additional diagrams with new particles running in the loops.

Dimension-6 effective interactions involving a Higgs and two gauge bosons can be expressed in terms of the following gauge-invariant operators $[18,19]$ :

$$
\begin{aligned}
\mathcal{O}_{B B} & =\frac{f_{B B}}{\Lambda^{2}} \Phi^{\dagger} \hat{B}_{\mu \nu} \hat{B}^{\mu \nu} \Phi \\
\mathcal{O}_{W W} & =\frac{f_{W W}}{\Lambda^{2}} \Phi^{\dagger} \hat{W}_{\mu \nu} \hat{W}^{\mu \nu} \Phi \\
\mathcal{O}_{B} & =\frac{f_{B}}{\Lambda^{2}} D_{\mu} \Phi^{\dagger} \hat{B}^{\mu \nu} D_{\nu} \Phi \\
\mathcal{O}_{W} & =\frac{f_{W}}{\Lambda^{2}} D_{\mu} \Phi^{\dagger} \hat{W}^{\mu \nu} D_{\nu} \Phi
\end{aligned}
$$

Where

$$
\begin{aligned}
B_{\mu \nu} & =\partial_{\mu} B_{\nu}-\partial_{\nu} B_{\mu} \\
\hat{B}_{\mu \nu} & =i \frac{g \tan \theta_{w}}{2} B_{\mu \nu}
\end{aligned}
$$

and

$$
\begin{aligned}
W^{a}{ }_{\mu \nu} & =\partial_{\mu} W^{a}{ }_{\nu}-\partial_{\nu} W_{\mu}^{a}-g \epsilon^{a b c} W_{\mu}^{b} W_{\mu}^{c} \\
\hat{W}_{\mu \nu} & =i \frac{g}{2} \sigma^{a} W^{a}{ }_{\mu \nu}
\end{aligned}
$$

These constitute the most general set of dimension- 6 effective operators which give rise to the $h \gamma \gamma$ and $h Z \gamma$ vertices. $\mathcal{O}_{B}$ and $\mathcal{O}_{W}$ contribute only to the $h Z \gamma$ vertex but not to $h \gamma \gamma$, since the component of Higgs doublet that acquires a vacuum expectation value (vev) has no electromagnetic charge. Furthermore, there is the operator $\mathcal{O}_{B W}$ which is of the form $\frac{f_{B W}}{\Lambda^{2}} \Phi^{\dagger} \hat{B}_{\mu \nu} \hat{W}^{\mu \nu} \Phi$. However, it leads to tree-level mixing between $\gamma$ and $Z$, thereby altering the $Z$-mass eigenstate, while keeping the $W$ mass eigenstate unaffected. Consequently, this term breaks the custodial SU(2) and therefore must be highly suppressed in general. This operator is not considered in the present analysis.

The contribution of the operators $\mathcal{O}_{B B}, \mathcal{O}_{W W}, \mathcal{O}_{B}$ and $\mathcal{O}_{W}$ to the $h \gamma \gamma$ and $h Z \gamma$ vertices can be obtained from the equation (2.5). However, as has been already mentioned, we have chosen to express only that part of the contributions to the loop-induced amplitude, which is the consequence of new particles running in the loops. The part coming from loops induced by $\mathrm{W}$ and the standard model fermions are used in their already available forms [17], with appropriate scaling of couplings denoted by the various $\kappa$-factors. As will be further explained in section 4, contributions from loops can be separately described in terms of dimension-6 operators. This is especially so when the corresponding Wilson 


\begin{tabular}{|c|c|c|}
\hline & $h \rightarrow \gamma \gamma$ & $h \rightarrow Z \gamma$ \\
\hline \multirow{2}{*}{$\mathcal{O}_{B B}$} & $-i \mathcal{M}_{B B}=$ & $-i \mathcal{M}_{B B}=$ \\
& $4 \frac{f_{B B}}{\Lambda^{2}} \frac{g^{2}}{4} \sin ^{2} \theta_{w} v \times$ & $-4 \frac{f_{B B}}{\Lambda^{2}} \frac{g^{2}}{2} \frac{\sin ^{3} \theta_{w}}{\cos \theta_{w}} v \times$ \\
\hline \multirow{2}{*}{$\mathcal{O}_{W W}$} & $\left(k_{1} \cdot k_{2} g_{\mu \nu}-k_{1 \mu} k_{2 \nu}\right) \epsilon^{* \mu}\left(k_{2}\right) \epsilon^{* \nu}\left(k_{1}\right)$ & $\left(k_{1} \cdot k_{2} g_{\mu \nu}-k_{1 \mu} k_{2 \nu}\right) \epsilon^{* \mu}\left(k_{2}\right) \epsilon^{* \nu}\left(k_{1}\right)$ \\
& $-i \mathcal{M}_{W W}=$ & $-i \mathcal{M}_{W W}=$ \\
& $4 \frac{f_{W W}}{\Lambda^{2}} \frac{g^{2}}{4} \sin ^{2} \theta_{w} v \times$ & $4 \frac{f_{W W}}{\Lambda^{2}} \frac{g^{2}}{2} \sin \theta_{w} \cos \theta_{w} v \times$ \\
& $\left(k_{1} \cdot k_{2} g_{\mu \nu}-k_{1 \mu} k_{2 \nu}\right) \epsilon^{* \mu}\left(k_{2}\right) \epsilon^{* \nu}\left(k_{1}\right)$ & $\left(k_{1} \cdot k_{2} g_{\mu \nu}-k_{1 \mu} k_{2 \nu}\right) \epsilon^{* \mu}\left(k_{2}\right) \epsilon^{* \nu}\left(k_{1}\right)$ \\
\hline
\end{tabular}

Table 1. The part of the amplitudes for $h \rightarrow \gamma \gamma$ and $h \rightarrow Z \gamma$ coming from new particle loops, and expressed in terms of the dimension-6 operators $\mathcal{O}_{B B}$ and $\mathcal{O}_{W W}$.

\begin{tabular}{|c|c|c|}
\hline & $h \rightarrow \gamma \gamma$ & $h \rightarrow Z \gamma$ \\
\hline \multirow{2}{*}{$\mathcal{O}_{B}$} & 0 & $-i \mathcal{M}_{B}=$ \\
& & $-2 \frac{f_{B}}{\Lambda^{2}} \frac{g g_{z}}{2} \sin \theta_{w} v \times$ \\
\hline \multirow{2}{*}{$\mathcal{O}_{W}$} & 0 & $-i \mathcal{M}_{W}=$ \\
& & $2 \frac{f_{W}}{\Lambda^{2}} \frac{g g_{z}}{4} \sin \theta_{w} v \times$ \\
$\left(k_{1} . k_{2} g_{\mu \nu}-k_{1 \mu} k_{2 \nu}\right) \epsilon^{* \mu}\left(k_{2}\right) \epsilon^{* \nu}\left(k_{1}\right)$ \\
\hline
\end{tabular}

Table 2. The part of the amplitudes for $h \rightarrow Z \gamma$ coming from new particle loops, and expressed in terms of the dimension-6 operators $\mathcal{O}_{B}$ and $\mathcal{O}_{W}$.

coefficients depend on gauge-invariant masses only. We apply this method to scenarios with additional scalars only, whose mass terms are gauge-invariant to start with. More will be said on this in section $4 .^{2}$

The parts of the contributions from the effective operators $\mathcal{O}_{B B}$ and $\mathcal{O}_{W W}$ are given in table. 1 while similar contributions from $\mathcal{O}_{B}$ and $\mathcal{O}_{W}$ are given in table. $2{ }^{3}$ We show in figure 1, the Feynman diagrams contributing to the decays $h \rightarrow \gamma \gamma$ and $h \rightarrow Z \gamma$, including those with scaled contribution of SM and those expressed in terms of loop induced dimension- 6 operators.

Rather precise results on $h \rightarrow \gamma \gamma$ are already available [1,20,21], and are usable in global fits that guide one to allowed values of the parameters invoked here. Similar results on $h \rightarrow Z \gamma$, dependent on leptonic decays of $Z$, are awaited till more luminosity accumulates. However, the upper limit on its production $\times$ branching ratio [22] available at any stage has served as a useful constraining factor, when it comes to obtaining the

\footnotetext{
${ }^{2}$ This analysis is legitimate at the one-loop level, where there is no interplay of SM and BSM loops. Although the effective operators in principle encapsulate contribution at all orders, it has been assumed that the dominant contribution comes at the one-loop level. Thus the two parts, namely those from the scaled SM loops and the ones from new particle contributions, can be taken to be of the same order of perturbation.

${ }^{3}$ We proved on the basis of the ansatz that the contribution to $h \rightarrow \gamma \gamma$ and $h \rightarrow Z \gamma$ from loops comprising new particles, which are interrelated by $\mathrm{SU}(2)_{L} \times \mathrm{U}(1)_{Y}$, are expressible as shown in the tables. This is possible only if the Wilson coefficients are insensitive to $m_{Z}$ and therefore the EWSB scale. This ansatz is justified in section 4 .
} 

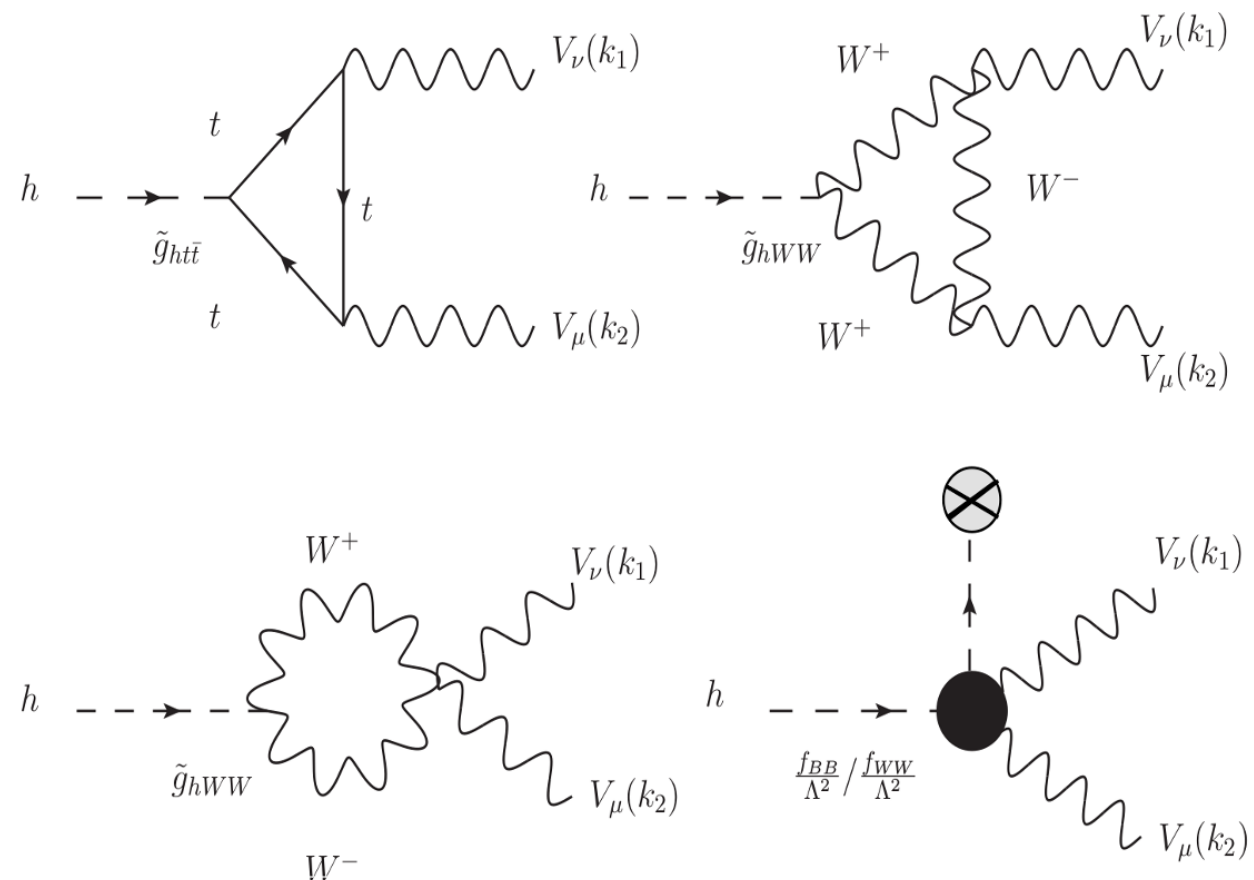

Figure 1. Feynman diagrams for $h \rightarrow \gamma \gamma$ and $h \rightarrow Z \gamma$ in the most general situation. Contributions mediated by fields other than those in SM are lumped in the blob.

statistically favored ranges of scaling factors as well as Wilson coefficients. This will be discussed in greater detail in section 4 .

The role and usefulness of higher-dimensional operators in dealing with unknown physics in Higgs phenomenology have already been discussed in the literature [2-13, 23]. For example, in [23], ways towards improved understanding on the dimension- $6 h V V$ operators in the high luminosity run of the LHC have been suggested. The utility of various ratios of signal strengths in different channels has figured in discussions found there. One such ratio, namely that of the signal strengths in the $\gamma \gamma$ and $Z \gamma$ channels, has been adopted as a component of the present analysis.

\section{The global fit}

After parametrizing new physics effects in the manner discussed above, we investigate the region of parameter space favored by the 8 and $13 \mathrm{TeV}$ results at the LHC. Our parameter space is eight-dimensional, spanned by the four scale factors $\kappa_{V}, \kappa_{t}, \kappa_{b}$ and $\kappa_{\tau}$ (which parametrize the modification of SM tree-level $h V V, h t \bar{t}, h b \bar{b}, h \tau \bar{\tau}$ couplings) and $f_{B B}, f_{W W}, f_{B}$ and $f_{W}$, which are the Wilson coefficients in the dimension- $6 h V V$ operators. In order to constrain new physics from experimental data, one needs to construct the likelihood function. This in principle is a non-trivial task, as experiment only provides the best fit and the $1 \sigma$ interval, but not the full likelihood function. However, assuming the 
measurements to be Gaussian, the Log-likelihood can be written as

$$
-2 \log L(\mu)=\left(\frac{\mu-\hat{\mu}}{\Delta \mu}\right)^{2}
$$

Where $\hat{\mu}$ is the experimental best fit value of the measured signal strength in some channel and $\Delta \mu$ is the corresponding standard deviation. If there are more than one independent measurements, then the full likelihood is a product of individual likelihoods. Consequently the combined $\chi^{2}$ is

$$
L(\mu)=\prod_{i=1}^{n} L_{i}(\mu) \Rightarrow \chi^{2}(\mu)=\sum_{i=1}^{n} \chi_{i}^{2}(\mu)=\sum_{i=1}^{n}\left(\frac{\mu-\hat{\mu}_{i}}{\Delta \mu_{i}}\right)^{2} .
$$

We would like to emphasize that the combined $\chi^{2}$ computation will follow equation (3.2) only when all the experimental measurements denoted by subscript $i$, are independent of each other. But in reality that assumption does not always hold. Various experimental searches share same systematic uncertainties and thus some correlation may exist between them. For example, different production channels leading to the same final can develop such correlation owing to misidentification of the production processes. Under the Gaussian approximation these correlations affect the Log-likelihood function in the following manner.

$$
-2 \log L(\mu)=\chi^{2}(\mu)=\left(\mu-\hat{\mu}_{i}\right)^{T} C_{i j}^{-1}\left(\mu-\hat{\mu}_{j}\right) .
$$

Where $C^{-1}$ is the inverse of the covariance matrix $C_{i j}=\operatorname{cov}\left(\hat{\mu}_{i}, \hat{\mu}_{j}\right)$ In the case where the measurements are independent, the covariance matrix is diagonal and $C_{i i}^{-1}$ will denote the variance $\sigma_{i}^{2}$. The correlations between gluon fusion and vector-boson fusion production for each of the major Higgs decay channels are found in [1, 20, 21, 24-27]. We extract the elements of the covariance matrices from those ellipses and calculate the combined $\chi^{2}$ for each point of the parameter space. This $\chi^{2}$ is then minimized with the help of the package MCMC [28], wherefrom one obtains the region allowed by the experimental data at the 1 -and $2 \sigma$ levels.

All 8 and $13 \mathrm{TeV}$ results from ATLAS and CMS available so far are included in our global fits. We thus take into account the $h \rightarrow \gamma \gamma, Z Z, W W, b \bar{b}$ and $\tau \bar{\tau}$ data from ATLAS and CMS at $8 \mathrm{TeV}$ with $20 \mathrm{fb}^{-1}$ integrated luminosity and $h \rightarrow \gamma \gamma, Z Z, W W, \tau \bar{\tau}$ from CMS $13 \mathrm{TeV}$ data with $36 \mathrm{fb}^{-1}$ integrated luminosity and $h \rightarrow \gamma \gamma, Z Z$ from ATLAS $13 \mathrm{TeV}$ data with $36 \mathrm{fb}^{-1}$ integrated luminosity. These details are presented in table. 3 .

We calculate the signal strengths $\mu=\sigma_{i} \times B R_{j}$, where the indices $i$ and $j$ indices denote respectively the production and decay channels of Higgs boson. One then proceeds to obtain $\chi_{\min }^{2}$, based on the experimental best-fit values and the corresponding error intervals, whence one arrives at plots showing the hypersurfaces allowed at the 1 -and $2 \sigma$ levels. Projections of these hypersurfaces into various two-parameter subspaces are shown in figures 2, 3 and 4, where all remaining parameters have been marginalized.

Figure 2 includes $2 \sigma$ contours in various pairs of the scaling parameters $\kappa$. The quadratic nature of the dependence of $\chi^{2}$ on the $\kappa$ 's results in the symmetric nature of the contours. 

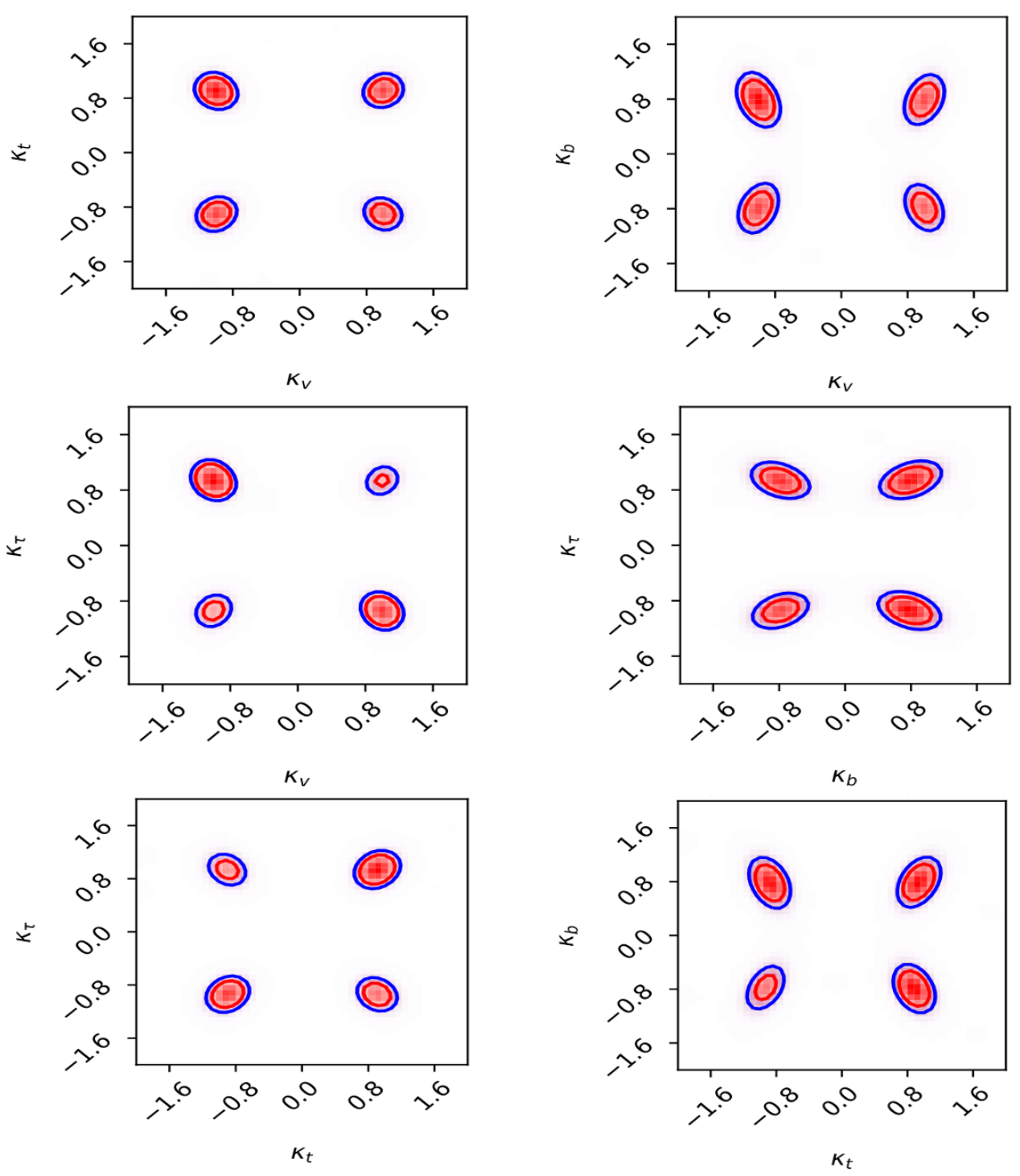

Figure 2. Allowed regions at $1 \sigma$ (red) and $2 \sigma$ (blue) levels in the parameter space of scale factors.

In figure 3 we show correlation between the $\kappa$ 's and the Wilson coefficients $f_{B B}$ and $f_{W W} \cdot f_{B}$ and $f_{W}$ contribute to $h \rightarrow Z \gamma$ but not $h \rightarrow \gamma \gamma$, since only the neutral component of the Higgs doublet acquires a vev. Therefore, they are not constrained by the observed signal strengths, but have only upper limits from the non-observation of the $Z \gamma$ final state so far.

Figure 4 (left) shows the $2 \sigma$ region in the $f_{B B^{-}} f_{W W}$ plane. There is a direction in this plane, where the signal strength in the $h \rightarrow \gamma \gamma$ channel will always be satisfied, as the contribution to the diphoton final state depends on $f_{B B}+f_{W W}$. This is evident from table. 1. On the other hand, the process $h \rightarrow Z \gamma$ receives contribution via the combination $\tan ^{2} \theta_{W} f_{B B}-f_{W W}$, thus indicating a region in the same place when the latter process fails to be restrictive. It is only from a combined imposition of both constraints that one 

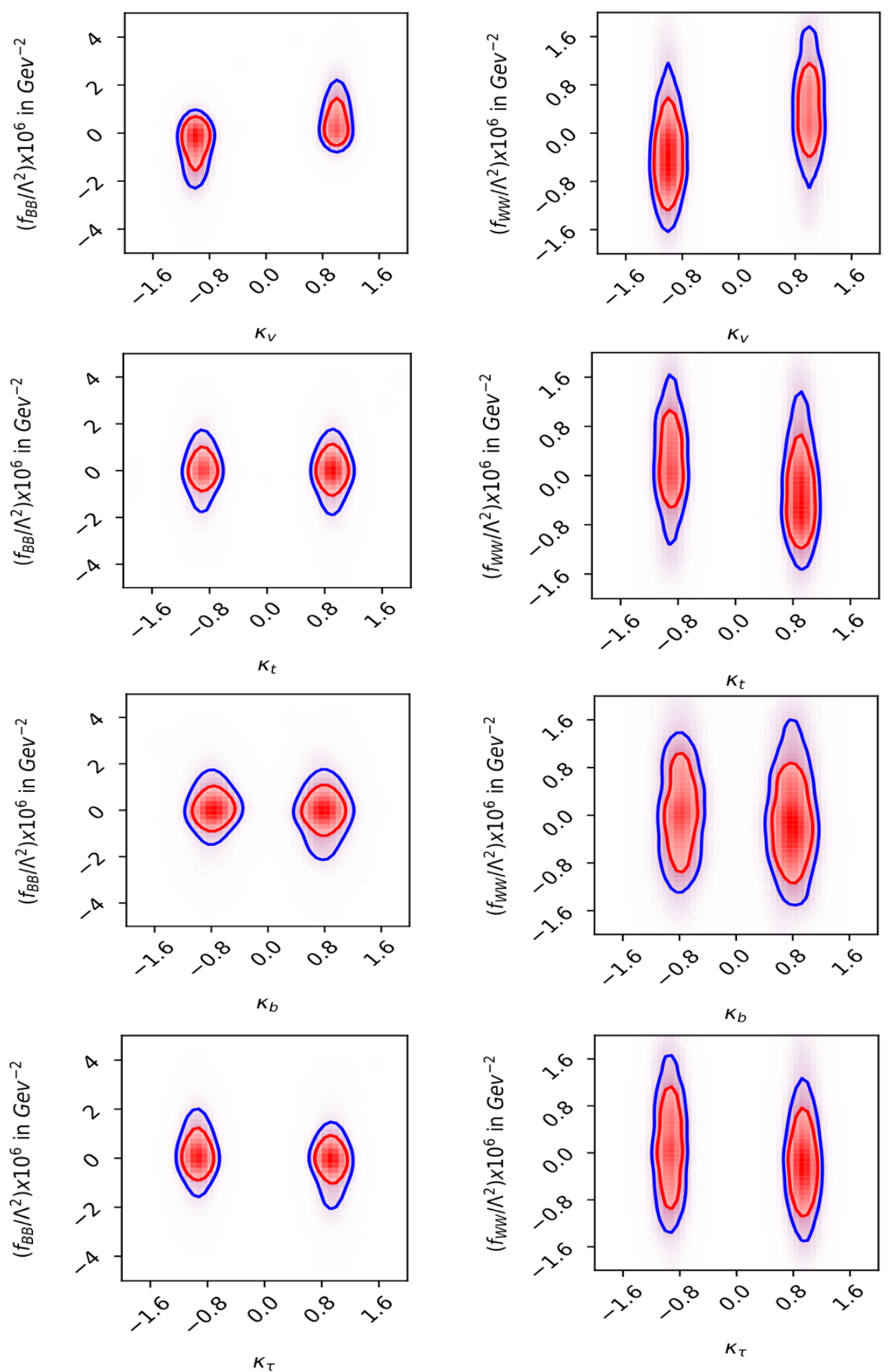

Figure 3. Allowed regions at $1 \sigma$ (red) and $2 \sigma$ (blue) levels in the parameter space of scale factors and dimension-6 couplings. 


\begin{tabular}{|c|c|c|c|}
\hline Channel & Experiment & Energy (GeV) & Luminosity \\
\hline$h \rightarrow \gamma \gamma$ & ATLAS + CMS [1] & $8 \mathrm{TeV}$ & $19.5 \mathrm{fb}^{-1}$ \\
\hline$h \rightarrow Z Z$ & ATLAS + CMS [1] & $8 \mathrm{TeV}$ & $19.5 \mathrm{fb}^{-1}$ \\
\hline$h \rightarrow W W$ & ATLAS + CMS [1] & $8 \mathrm{TeV}$ & $19.5 \mathrm{fb}^{-1}$ \\
\hline$h \rightarrow b \bar{b}$ & ATLAS + CMS [1] & $8 \mathrm{TeV}$ & $19.5 \mathrm{fb}^{-1}$ \\
\hline$h \rightarrow \tau \bar{\tau}$ & ATLAS + CMS [1] & $8 \mathrm{TeV}$ & $19.5 \mathrm{fb}^{-1}$ \\
\hline$h \rightarrow \gamma \gamma$ & CMS [20] & $13 \mathrm{TeV}$ & $36 \mathrm{fb}^{-1}$ \\
\hline$h \rightarrow Z Z$ & CMS [24] & $13 \mathrm{TeV}$ & $36 \mathrm{fb}^{-1}$ \\
\hline$h \rightarrow W W$ & CMS [25] & $13 \mathrm{TeV}$ & $15.2 \mathrm{fb}^{-1}$ \\
\hline$h \rightarrow \tau \bar{\tau}$ & CMS [26] & $13 \mathrm{TeV}$ & $36 \mathrm{fb}^{-1}$ \\
\hline$h \rightarrow \gamma \gamma$ & ATLAS [21] & $13 \mathrm{TeV}$ & $36 \mathrm{fb}^{-1}$ \\
\hline$h \rightarrow Z Z$ & ATLAS [27] & $13 \mathrm{TeV}$ & $36 \mathrm{fb}^{-1}$ \\
\hline
\end{tabular}

Table 3. Listing of the Higgs signal strength measurements at LHC at 8 and $13 \mathrm{TeV}$.
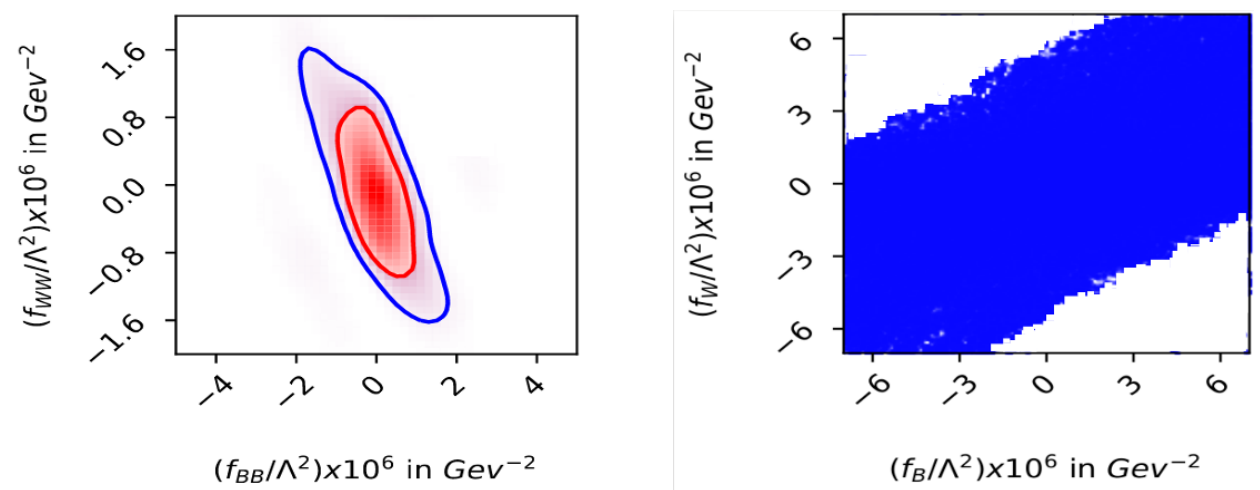

Figure 4. (left) Allowed regions at $1 \sigma(\mathrm{red})$ and $2 \sigma$ (blue) levels in the $f_{B B^{-}} f_{W W}$ plane and (right) allowed region at $2 \sigma$ level in the $f_{B^{-}} f_{W}$ plane.

obtains a closed region in the $f_{B B^{-}} f_{W W}$, as can be seen in figure 4 (left). Figure 4 (right) shows the favored regions in the $f_{B^{-}} f_{W}$ plane. As $f_{B}$ and $f_{W}$ get contribution only from the upper limit of $h \rightarrow Z \gamma$ process [22], we do not get a closed region in this case.

\section{Extended Higgs models and dimension-6 operators}

Various new physics models predict extended electroweak symmetry breaking sectors. It is naturally of interest to link the model-independent analysis presented above to specific theoretical scenarios. It has to be remembered that most of these scenarios are strongly constrained by electroweak precision data [29] as well as observed signal strengths of the already discovered Higgs [1, 20, 21, 24-27]. From the standpoint of uncovering new theoretical structures, it is therefore a challenge to extract the tiniest tractable departures from SM effects. These may occur both as multiplicative alteration of interaction strength(s) and, perhaps more significantly, via higher-dimensional operators. With this in view, we 
now translate the results of the previous section to those pertaining to extended Higgs models, taking into account the additional constraints that connect model parameters in each case.

The simplest set of extensions consists in two Higgs doublet models (2HDM) [17, 30]. Motivated and largely popularized by the minimal supersymmetric standard Model (MSSM), such scenarios are of interest independently of supersymmetry. They are of interest due to their phenomenological richness but also, for example, by the observation that $2 \mathrm{HDM}$ allows a stable electroweak vacuum all the way to the Planck scale without the aid of any new physics [31].

At the same time, scalars in higher SU(2) representations are often helpful in understanding basic issues such as neutrino mass generation. Introduction of a $Y=2$ complex $\mathrm{SU}(2)$ triplet enables the Type II seesaw mechanism to yield Majorana masses for neutrinos [32]. The strong constraint on the triplet vev from the $\rho$-parameter is not only consistent with such mechanism but in fact justifies the smallness of neutrino masses [33-36]. Furthermore, some model-building efforts to connect neutrino masses with their mixing angles motivates Type II seesaw with two triplets instead of one [37].

We analyze some of these models here, with reference to the model-independent approach of the previous section. All scenarios discussed here predict one or more, singly or doubly, charged Higgs bosons. These charged scalars should contribute to loop-induced decays of the SM-like Higgs, namely, $h \rightarrow \gamma \gamma, h \rightarrow Z \gamma$. The corresponding decay widths deviate from the SM predictions due to (a) scaling of the $h W W, h t \bar{t}$ vertices, and (b) the additional contributions from diagrams of the kind shown in figure 5, where the $V^{\mu}$ and $V^{\nu}$ in the external legs stand for $\gamma \gamma$ or $\gamma Z$. While the former lead to the factors denoted by $\kappa$, the latter are parametrized in our approach by dimension- 6 operators. The difference compared to the model-independent case is that the two classes of modifiers are related once model parameters are specified. Such mutual dependence has to be taken into account in each particular situation, as will be discussed section 4.1 onwards.

The contribution to the amplitude from the charged scalar loops for $h \rightarrow \gamma \gamma$ is of the form $[17]$

$$
-i \mathcal{M}=C_{\text {vertex }} \times\left(k_{1} \cdot k_{2} g_{\mu \nu}-k_{1 \mu} k_{2 \nu}\right) \epsilon^{* \mu}\left(k_{2}\right) \epsilon^{* \nu}\left(k_{1}\right) \times A_{H}(\gamma \gamma)\left(m_{H^{ \pm}}\right)
$$

While that for $h \rightarrow Z \gamma$ is [17]

$$
-i \mathcal{M}=\tilde{C}_{\text {vertex }} \times\left(k_{1} \cdot k_{2} g_{\mu \nu}-k_{1 \mu} k_{2 \nu}\right) \epsilon^{* \mu}\left(k_{2}\right) \epsilon^{* \nu}\left(k_{1}\right) \times A_{H}(Z \gamma)\left(m_{H^{ \pm}}, m_{Z}\right)
$$

Where $C_{\text {vertex }}$ and $\tilde{C}_{\text {vertex }}$ are the vertex factors for $h \rightarrow \gamma \gamma$ and $h \rightarrow Z \gamma$ in the two cases, while $A_{H}(\gamma \gamma)$ and $A_{H}(Z \gamma)$ are the loop integrals for $h \rightarrow \gamma \gamma$ and $h \rightarrow Z \gamma$ respectively.

The current direct search and B-physics observables do not put strong limits on charged Higgs masses except in very specific models. Therefore, the charged scalars need not always be much heavier than the electroweak scale, and can easily be just a few hundred GeV in mass.

Therefore, while parametrizing the amplitudes in terms of gauge invariant effective operators, one may still have 'effective' suppression scales $\gtrsim \mathrm{TeV}$. The combination of 


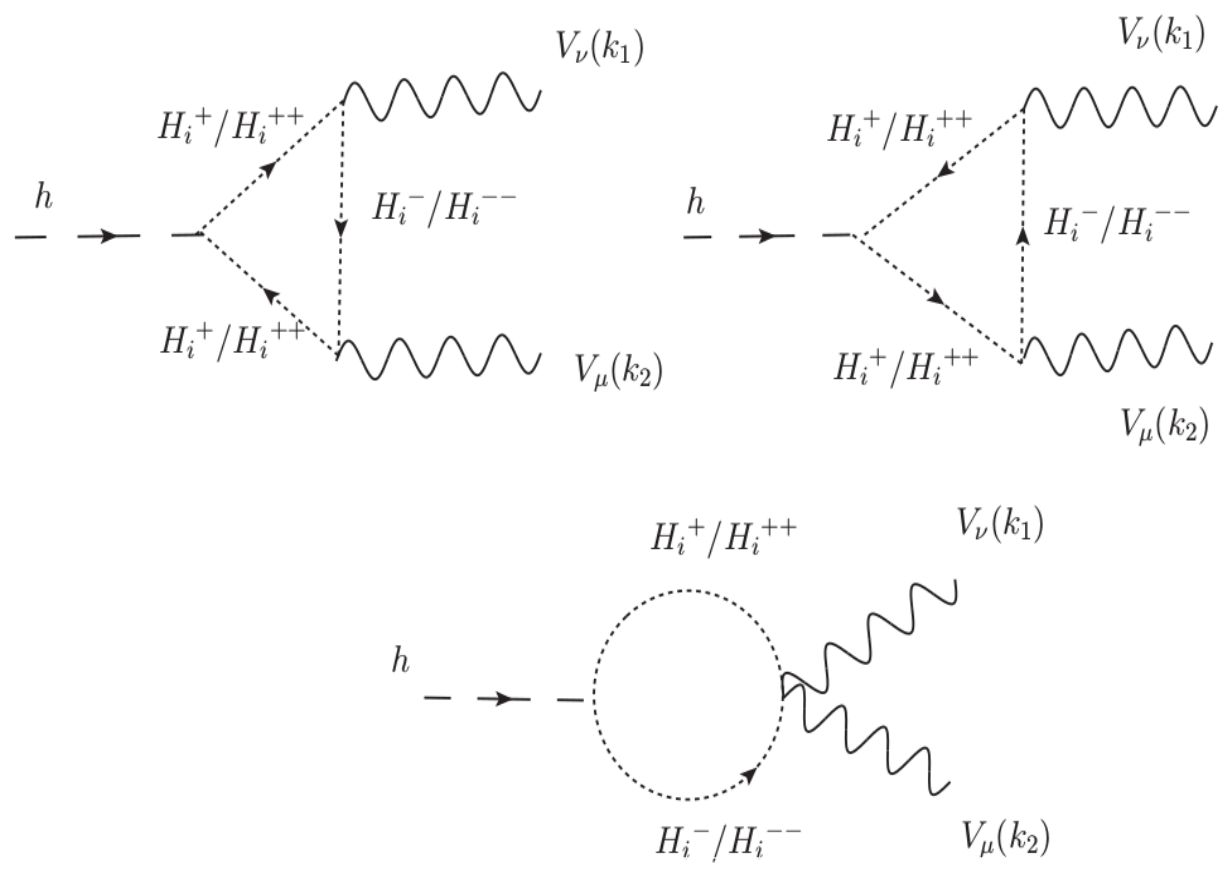

Figure 5. Additional scalar loop contributions to $h \rightarrow \gamma \gamma$ and $h \rightarrow Z \gamma$.

Wilson coefficients contributing to the decay amplitudes, evaluated at the EWSB scale, may in principle have a functional dependence on $m_{\mathrm{EWSB}} \simeq m_{Z}$ due to renormalization group (RG) running. However, this dependence is rather weak in general, unless one has strongly coupled high-scale dynamics.

In the cases under consideration here, the loop amplitude for $h \rightarrow \gamma \gamma$ does not involve $m_{Z}$, and the charged scalar masses arises from $\mathrm{SU}(2)_{L} \times \mathrm{U}(1)_{Y}$ invariant terms. Thus there is no dependence on $m_{\mathrm{EWSB}}$ there. One would thus expect the same dependence (or lack of it) in the amplitude for $h \rightarrow Z \gamma$ unless there are highly fine-tuned boundary conditions in the RG running of parameters. A natural way of establishing consistency between the two amplitudes, therefore, is to have no $m_{Z}$-dependence in the loop amplitude for $h \rightarrow Z \gamma$ as well. As one can see from figure 6 , this is indeed the case here; the loop integrals are insensitive to an 'artificial' variation of $m_{Z}$ so long as the charged Higgs(es) circulating in the loop stay above that mass. It is, therefore, legitimate to encapsulate the contributions to these loop amplitudes as Wilson coefficients of dimension-6 gauge-invariant operators, so long as the charged scalars responsible for these amplitude are at least a factor of two heavier than the $Z$.

For reasons already mentioned, we adopt four kinds of $2 \mathrm{HDM}$ and also scenarios with one and two scalar triplets to illustrate our approach. We take them up in turn in the following subsections. We mention here that in the analysis that follows, we have marginalized over the parameters $f_{B}$ and $f_{W}$. These two parameters get constrained only from the upper limit on $h \rightarrow Z \gamma$ decay width. Thus their role in this analysis is to stay within the boundary defined by this upper limit; no further information can be obtained at present due to absence of any positive result. 


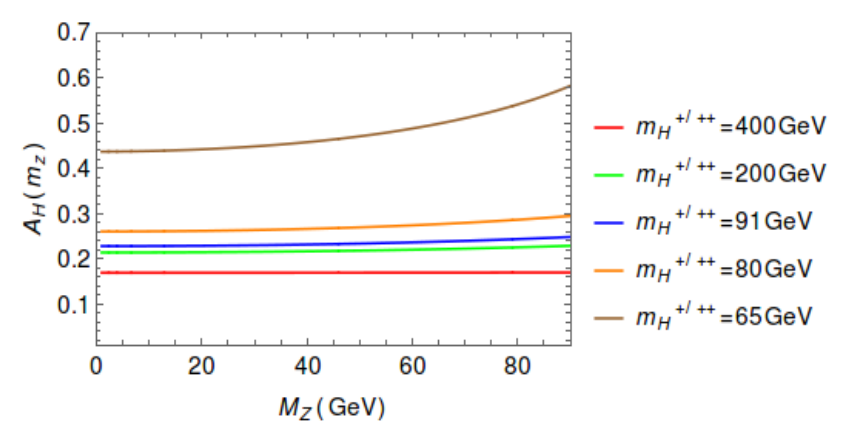

Figure 6. Dependence of the additional scalar loop integral on the $Z$ boson mass in $h \rightarrow Z \gamma$.

\subsection{Two Higgs doublet models}

The most general $2 \mathrm{HDM}$ potential contains fourteen parameters $[17,30]$. It can have both CP-conserving and CP-violating terms in principle. However, we keep the analysis simple by assuming no $\mathrm{CP}$-violation in the Higgs sector. The most general $\mathrm{CP}$-conserving potential with two Higgs doublets $\Phi_{1}$ and $\Phi_{2}$ is

$$
\begin{aligned}
V= & m_{11}^{2} \Phi_{1}^{\dagger} \Phi_{1}+m_{22}^{2} \Phi_{2}^{\dagger} \Phi_{2}-m_{12}^{2}\left(\Phi_{1}^{\dagger} \Phi_{2}+\Phi_{2}^{\dagger} \Phi_{1}\right)+\frac{\lambda_{1}}{2}\left(\Phi_{1}^{\dagger} \Phi_{1}\right)^{2}+\frac{\lambda_{2}}{2}\left(\Phi_{2}^{\dagger} \Phi_{2}\right)^{2} \\
& +\lambda_{3} \Phi_{1}^{\dagger} \Phi_{1} \Phi_{2}^{\dagger} \Phi_{2}+\lambda_{4} \Phi_{1}^{\dagger} \Phi_{2} \Phi_{2}^{\dagger} \Phi_{1}+\frac{\lambda_{5}}{2}\left[\left(\Phi_{1}^{\dagger} \Phi_{2}\right)^{2}+\left(\Phi_{2}^{\dagger} \Phi_{1}\right)^{2}\right]
\end{aligned}
$$

where all coefficients are real. The minimum of this potential is obtained when the neutral components of $\Phi_{1}$ and $\Phi_{2}$ acquire vev $v_{1}$ and $v_{2}$ respectively.

$$
\begin{aligned}
\left\langle\phi_{1}\right\rangle_{0} & =\frac{1}{\sqrt{2}}\left(\begin{array}{c}
0 \\
v_{1}
\end{array}\right) \\
\left\langle\phi_{2}\right\rangle_{0} & =\frac{1}{\sqrt{2}}\left(\begin{array}{c}
0 \\
v_{2}
\end{array}\right) \\
\phi_{a}(x) & =\frac{1}{\sqrt{2}}\left(\begin{array}{c}
\phi_{a}^{+}(x) \\
v_{a}+\sigma_{a}(x)+i \eta_{a}(x)
\end{array}\right)
\end{aligned}
$$

Where $a=1,2$. After substituting this form of $\Phi_{1,2}$ in equation (4.3), we obtain the mass matrices for the charged and neutral Higgs bosons. Their diagonalization yields the masses of the charged scalar, two neutral scalars as well as the neutral pseudoscalar. The charged scalar mass in particular is given by

$$
m_{H^{ \pm}}^{2}=\left[m_{12}^{2} /\left(v_{1} v_{2}\right)-\lambda_{4}-\lambda_{5}\right]\left(v_{1}^{2}+v_{2}^{2}\right)
$$

These masses, together with the neutral scalar mixing angle $\alpha$ and charged and pseudoscalar mixing angle $\beta\left(\tan \beta=\frac{v_{2}}{v_{1}}\right)$ constitute the set of free parameters in this model.

The Yukawa sector is what mainly distinguishes one subclass of 2HDM from another. These subclasses have developed largely from different discrete symmetries postulated to 
address the issue of avoiding tree-level flavor changing neutral currents (FCNC). The most general Yukawa Lagrangian in a 2HDM can be expressed as

$$
\begin{aligned}
\mathcal{L}= & y_{i j}^{1} \bar{Q}_{i L} \Phi_{1} d_{j R}+y_{i j}^{2} \bar{Q}_{i L} \bar{\Phi}_{1} u_{j R}+y_{i j}^{3} \bar{Q}_{i L} \Phi_{2} d_{j R}+y_{i j}^{4} \bar{Q}_{i L} \bar{\Phi}_{2} u_{j R} \\
& +y_{i j}^{5} \bar{L}_{i L} \Phi_{1} e_{j R}+y_{i j}^{6} \bar{L}_{i L} \Phi_{2} e_{j R}
\end{aligned}
$$

FCNC constraints discourage the coupling of both doublets with $T_{3}=+$ and fermions. The most popular way to circumvent this problem is to impose a $Z_{2}$ symmetry, thereby prohibiting some of the terms above. Different combinations of the $Z_{2}$ quantum numbers of the fields present in equation (4.8) lead to at least four types of $2 \mathrm{HDM}$, which are brought under our analysis in turn.

In the subsequent discussions we will mainly focus on Higgs decay to $\gamma \gamma$ and $Z \gamma$ channels, which is dominated by top-and $W$-loops in SM. In all the variants of 2HDM, the charged Higgs boson $\left(H^{ \pm}\right)$contributes additionally to this processes at the one-loop level. The modification of the $W$ and top loop over and above their SM values and the additional charged Higgs loop contribution is given in detail in appendix A.

\subsubsection{Type-I 2HDM}

Here all fermions are assumed to couple to the same doublet, so that equation (4.8) reduces to

$$
\mathcal{L}_{\text {Yukawa }}=y_{i j}^{1} \bar{Q}_{i L} \Phi_{2} d_{j R}+y_{i j}^{2} \bar{Q}_{i L} \bar{\Phi}_{2} u_{j R}+y_{i j}^{5} \bar{L}_{i L} \Phi_{2} e_{j R}
$$

This can be achieved by imposing the discrete symmetry on the $\mathcal{L}_{\text {Yukawa }}, \Phi_{1} \rightarrow-\Phi_{1}$. The couplings of the fermions with the SM-like Higgs boson in this case are

$$
\begin{aligned}
C_{h t \bar{t}} & =\frac{\cos \alpha}{\sin \beta} \times C_{h t \bar{t}}^{\mathrm{S} M} \\
C_{h b \bar{b}} & =\frac{\cos \alpha}{\sin \beta} \times C_{h b \bar{b}}^{\mathrm{S} M} \\
C_{h \tau \bar{\tau}} & =\frac{\cos \alpha}{\sin \beta} \times C_{h \tau \bar{\tau}}^{\mathrm{S} M}
\end{aligned}
$$

while the gauge boson couplings are

$$
C_{h V V}=\sin (\beta-\alpha) \times C_{h V V}^{\mathrm{S} M}
$$

These define, in this case as well as the other types of $2 \mathrm{HDM}$, the corresponding $\kappa_{v}, \kappa_{t}$, $\kappa_{b}$ and $\kappa_{\tau}$. Here they all of the same value, denoted by the factor $\kappa_{f}$. Thus the departure from SM effects in data concerning the $125 \mathrm{GeV}$ scalar can be described here in terms of six parameters, namely, $\kappa_{v}, \kappa_{f}, f_{B B}, f_{W W}, f_{B}, f_{W}$. Following the same procedure as in section 2 and assuming all the six parameters to be independent, the $1 \sigma$ and $2 \sigma$ allowed regions for them are found to be as in figure 7 , which looks very similar to the corresponding contours in figures 2 and 4 .

The task now is to translate these into limits on the model parameter space. In order to do that, we note that in this model,

$$
\begin{aligned}
& \kappa_{v}=\sin (\beta-\alpha) \\
& \kappa_{f}=\frac{\sin \alpha}{\cos \beta}=\sin (\beta-\alpha)-\cos (\beta-\alpha) \tan \beta
\end{aligned}
$$



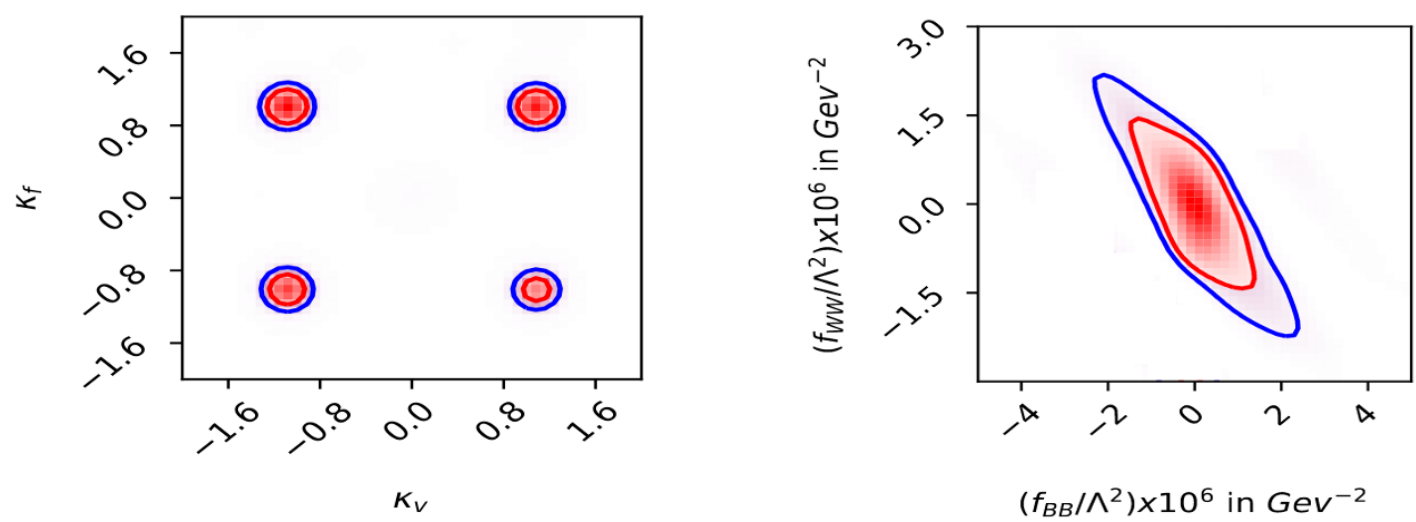

Figure 7. Allowed regions at $1 \sigma$ (red) and $2 \sigma$ (blue) levels in the parameter space of scale factors and dimension- 6 couplings in Type I 2HDM.

The top, bottom and $W$ loop contribution to $h \rightarrow \gamma \gamma$ will be modified by these scale factors. The charged Higgs loop contribution will be a function of $m_{H^{ \pm}}$multiplied by the quantity $g_{h H^{+} H-}$ which is given by

$$
g_{h H^{+} H^{-}}=\sin (\beta-\alpha)+\frac{\cos 2 \beta \sin (\beta+\alpha)}{2 \cos ^{2} \theta_{W}}
$$

At this point we draw the attention of the reader to figure 7 . Unlike in the modelindependent case, we find this time an interrelation of $f_{B B}, f_{W W}$ and $\kappa$, since $\alpha$ and $\beta$ enter into all of them. At the same time, there is a single $\kappa$ here, pointing again at a correlation among the scaling factors.

The fact that the top-quark coupling plays the most crucial role here in fitting the data shows little departure in the left panel of figure 7, as compared to the corresponding contour in figure 2. As for the right panel, while $f_{B B}, f_{W W}$ is correlated with $\kappa$, the availability of an extra parameter $m_{H^{ \pm}}$softens this correlation, since the entire $2 \sigma$ contour in $f_{B B}-f_{W W}$ plane will be allowed for some value of $m_{H^{ \pm}}$. That is why the right panel of figure 7 is very similar to the corresponding figure in figure 4 , modulo the fact that here is only $\kappa_{v}$ and $\kappa_{f}$ that are marginalized.

For the sake of simplicity in the analytical calculation we define a parameter which is a function of the charged Higgs mass:

$$
f\left(m_{H^{ \pm}}\right)=\tau f^{0}(\tau)
$$

where $\tau$ and $f^{0}(\tau)$ have been defined in equations (A.3) and (A.4). This function encapsulates the contribution of the charged Higgs loop integral.

Keeping the above discussion in mind, one arrives at the $1 \sigma$ and $2 \sigma$ contours in the $\sin (\beta-\alpha)-f\left(m_{H^{ \pm}}\right)$and $g_{h H^{+} H^{--}} f\left(m_{H^{ \pm}}\right)$planes in figure 8 (left) and (right) respectively. We mention here in the context of loop induced effects, $f\left(m_{H^{ \pm}}\right)$can only take positive values, as otherwise one ends up with an inadmissibly small charged Higgs mass (see equation (A.4)). 

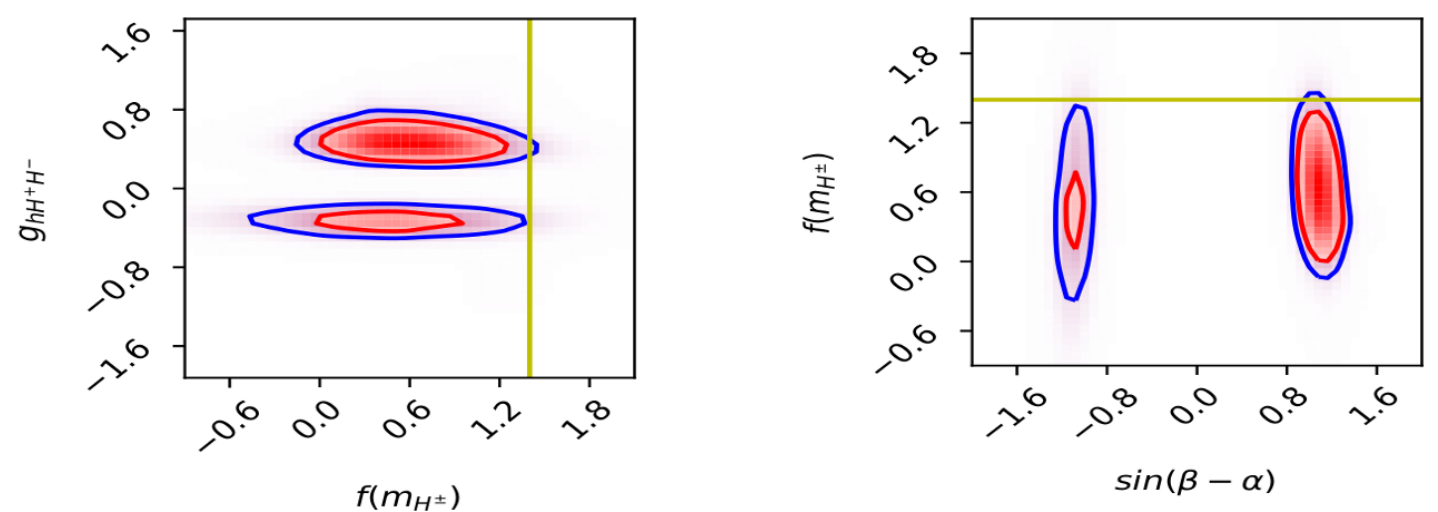

Figure 8. Allowed regions at $1 \sigma$ (red) and $2 \sigma$ (blue) levels in the parameter space of $f\left(m_{H^{ \pm}}\right)-$ $g_{h H^{+} H^{-}}$(left) and $\sin (\beta-\alpha)-f\left(m_{H^{ \pm}}\right)$(right) in Type I $2 \mathrm{HDM}$.

\subsubsection{Type-II 2HDM}

Another way to stay away from tree-level FCNC is to allow up-type quarks to couple to one doublet, and down-type quarks and leptons to couple to another. Under this assumption, the $\mathcal{L}_{\text {Yukawa }}$ becomes

$$
\mathcal{L}_{\text {Yukawa }}=y_{i j}^{1} \bar{Q}_{i L} \Phi_{1} d_{j R}+y_{i j}^{2} \bar{Q}_{i L} \bar{\Phi}_{2} u_{j R}+y_{i j}^{5} \bar{L}_{i L} \Phi_{1} e_{j R}
$$

This can be enforced by demanding that the $\mathcal{L}_{\text {Yukawa }}$ remains invariant under $\Phi_{1} \rightarrow-\Phi_{1}$ and $d_{R} \rightarrow-d_{R}$ and $e_{R} \rightarrow-e_{R}$.

The SM-like Higgs boson now has scaled interactions with SM fermions and gauge bosons given by

$$
\begin{aligned}
C_{h t \bar{t}} & =\frac{\cos \alpha}{\sin \beta} \times C_{h t \bar{t}}^{\mathrm{S} M} \\
C_{h b \bar{b}} & =-\frac{\sin \alpha}{\cos \beta} \times C_{h b \bar{b}}^{\mathrm{S} M} \\
C_{h \tau \bar{\tau}} & =-\frac{\sin \alpha}{\cos \beta} \times C_{h \tau \bar{\tau}}^{\mathrm{S} M} \\
C_{h V V} & =\sin (\beta-\alpha) \times C_{h V V}^{\mathrm{S} M}
\end{aligned}
$$

The top, bottom and $\tau$ couplings with Higgs are not modified in the same way any more. However, equation (4.17). implies

$$
\kappa_{\tau}=\kappa_{b}=\frac{\kappa_{t}^{2}-2 \kappa_{t} \kappa_{v}+1}{\kappa_{t}-\kappa_{v}}
$$

On using these relations, we can again reduce the 8-dimensional parameter space to a 6 -dimensional one, albeit via different constraints. The independent parameters are once more $\kappa_{v}, \kappa_{t}, f_{B B}, f_{W W}, f_{B}$ and $f_{W}$. The allowed parameter space at $1 \sigma$ and $2 \sigma$ regions. In $\kappa_{v^{-}} \kappa_{t}$ and $f_{B B^{-}} f_{W W}$ parameter space can be seen in figure 9 (left) and (right) respectively.

One now translates these limits into those on the model parameter space, using

$$
\begin{aligned}
\kappa_{v} & =\sin (\beta-\alpha) \\
\kappa_{t} & =\frac{\cos \alpha}{\sin \beta}=\sin (\beta-\alpha)-\cos (\beta-\alpha) \tan \beta
\end{aligned}
$$



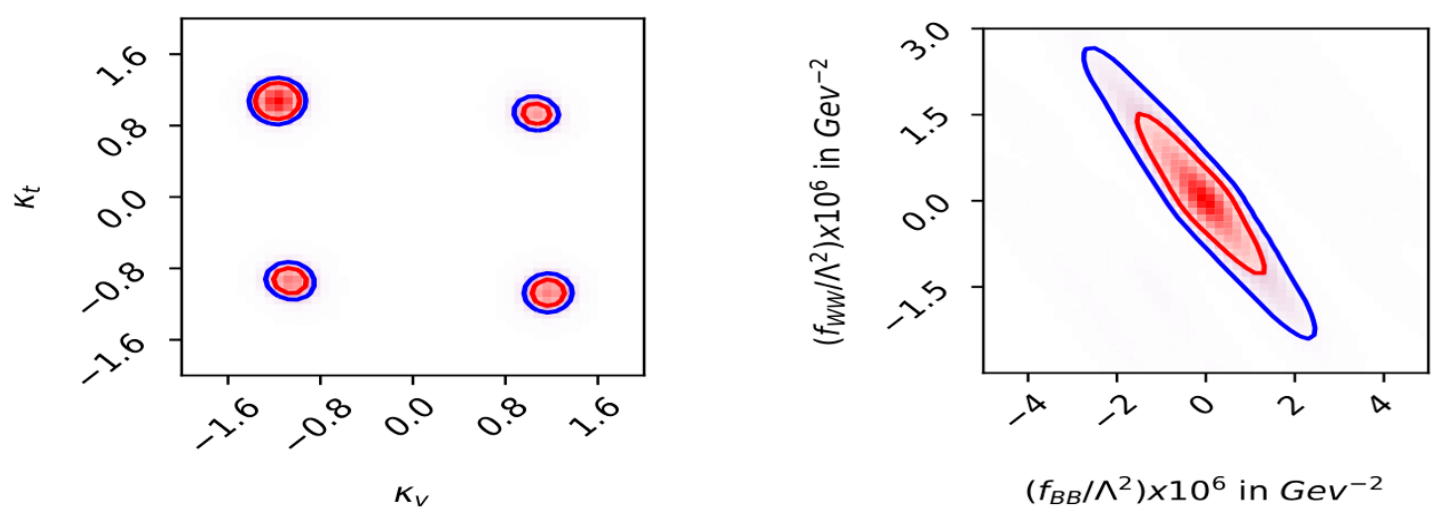

Figure 9. Allowed regions at $1 \sigma$ (red) and $2 \sigma$ (blue) levels in the parameter space of scale factors and dimension- 6 couplings in Type II 2HDM.
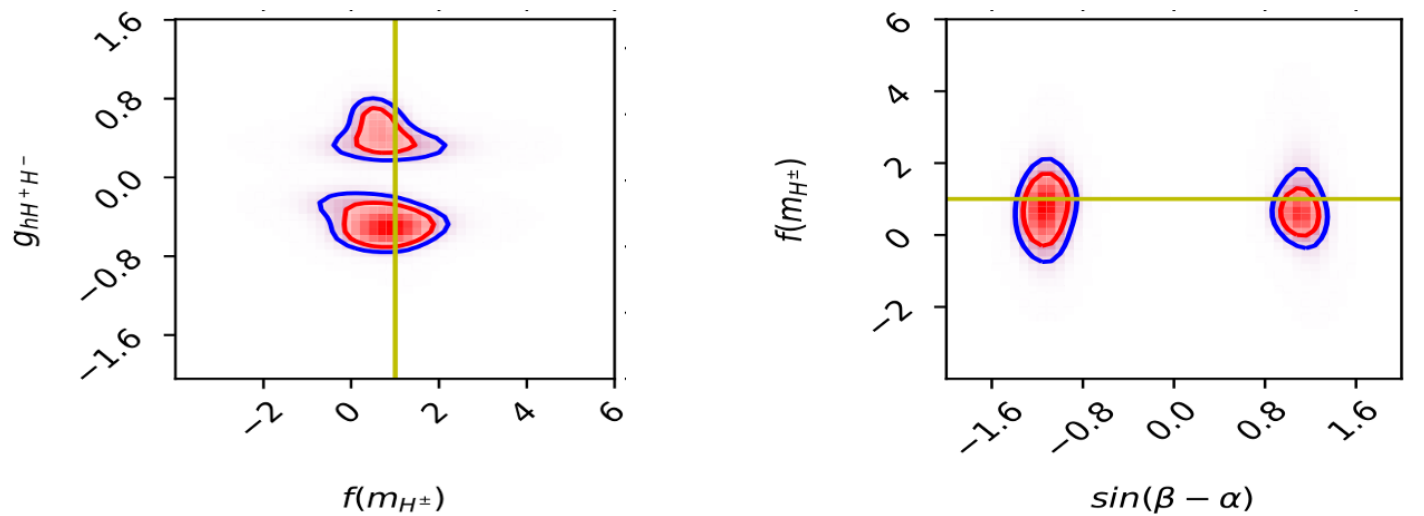

Figure 10. Allowed regions at $1 \sigma(\mathrm{red})$ and $2 \sigma$ (blue) levels in the parameter space of $f\left(m_{H^{ \pm}}\right)-$ $g_{h H^{+} H^{-}}$(left) and $\sin (\beta-\alpha)-f\left(m_{H^{ \pm}}\right)$(right) in Type II 2HDM.

$\kappa_{b}$ and $\kappa_{\tau}$ can be obtained following equations (4.17) and (4.18). The top, bottom and $W$ loop contribution to the decay $h \rightarrow \gamma \gamma$ will be modified by scale factors as described above. The new charged Higgs loop contribution, too, will depend on $\sin (\beta-\alpha), \tan \beta$ and $m_{H^{ \pm}}$as discussed in the previous subsection. A similar procedure as that for Type-I $2 \mathrm{HDM}$ leads to the contours in figure 10.

It should be noted that contours in the $\kappa_{v}-\kappa_{t}$ here exhibit more departure from the corresponding figure in figure 2 than is seen for Type-I 2HDM. This is because the correlated scale factors $\kappa_{b}$ and $\kappa_{\tau}$ have a stronger role to play here, as they make significant contributions to loop-induced Higgs decays for high values of $\tan \beta$, thus causing shifts in the allowed contours. In addition, the allowed region in the right panel of figure 10 suffers from stronger constraints than in the previous case, as the charged Higgs mass has a lower limit of $580 \mathrm{GeV}$ in Type-II 2HDM from the rare decay $b \rightarrow s \gamma$ [38-42].

\subsubsection{Lepton-specific 2HDM}

An alternative way is to allow the up-and down-type quarks to couple to one doublet and leptons to the other doublet. This can be achieved by imposing the symmetry of 

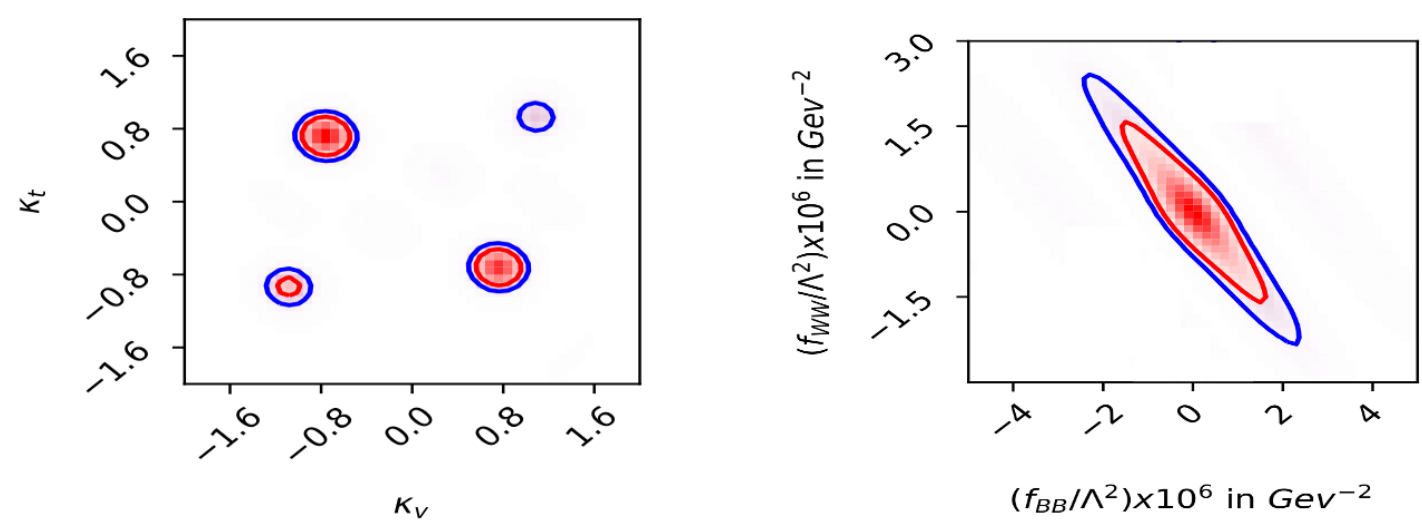

Figure 11. Allowed regions at $1 \sigma$ (red) and $2 \sigma$ (blue) levels in the parameter space of scale factors and dimension- 6 couplings in Lepton-specific 2HDM.

the Lagrangian under the discrete symmetry $\Phi_{1} \rightarrow-\Phi_{1}$ and $e_{R} \rightarrow-e_{R}$. $\mathcal{L}_{\text {Yukawa }}$ in this case becomes

$$
\mathcal{L}_{\text {Yukawa }}=y_{i j}^{1} \bar{Q}_{i L} \Phi_{2} d_{j R}+y_{i j}^{2} \bar{Q}_{i L} \bar{\Phi}_{2} u_{j R}+y_{i j}^{5} \bar{L}_{i L} \Phi_{1} e_{j R}
$$

In this scenario the scale factors which modify the SM Higgs coupling with fermions and gauge bosons, take the following forms:

$$
\begin{aligned}
C_{h t \bar{t}} & =\frac{\cos \alpha}{\sin \beta} \times C_{h t \bar{t}}^{\mathrm{SM}} \\
C_{h b \bar{b}} & =\frac{\cos \alpha}{\sin \beta} \times C_{h b \bar{b}}^{\mathrm{S} M} \\
C_{h \tau \bar{\tau}} & =-\frac{\sin \alpha}{\cos \beta} \times C_{h \tau \bar{\tau}}^{\mathrm{S} M} \\
C_{h V V} & =\sin (\beta-\alpha) \times C_{h V V}^{\mathrm{S} M}
\end{aligned}
$$

The up and down-type quark couplings thus get modified in the same manner, whereas the lepton coupling is different. Going back to our parametrization of new physics in the model-independent framework,

$$
\begin{aligned}
\kappa_{t} & =\kappa_{b} \\
\kappa_{\tau} & =\frac{\kappa_{t}^{2}-2 \kappa_{t} \kappa_{v}+1}{\kappa_{t}-\kappa_{v}}
\end{aligned}
$$

Our model independent 8-dimensional parameter space shrinks again to a 6 -dimensional parameter space spanned by $\kappa_{v}, \kappa_{t}, f_{B B}, f_{W W}, f_{B}, f_{W}$. The allowed parameter space at $1 \sigma$ and $2 \sigma$ levels is shown in figure 11 (left) in $\kappa_{v}-\kappa_{t}$ plane and in figure 11 (right) in $f_{B B^{-}} f_{W W}$ plane, from which one sets about translating these limits to those on the model parameter space.

$$
\begin{aligned}
\kappa_{v} & =\sin (\beta-\alpha) \\
\kappa_{t} & =\frac{\cos \alpha}{\sin \beta}=\sin (\beta-\alpha)-\cos (\beta-\alpha) \tan \beta
\end{aligned}
$$



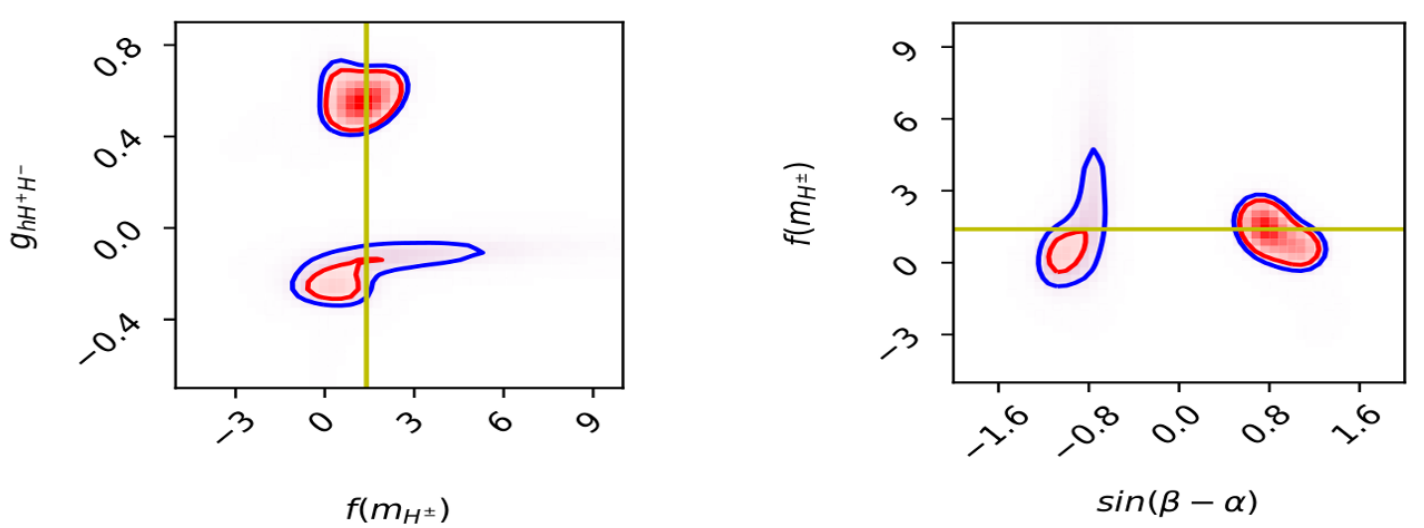

Figure 12. Allowed regions at $1 \sigma$ (red) and $2 \sigma$ (blue) levels in the parameter space of $f\left(m_{H^{ \pm}}\right)-$ $g_{h H^{+} H^{-}}$(left) and $\sin (\beta-\alpha)-f\left(m_{H^{ \pm}}\right)$(right) in Lepton-specific 2HDM.

Equating the loop contribution to $h \rightarrow \gamma \gamma$ from both the approaches, we plot the $1 \sigma$ and $2 \sigma$ contours in the $f\left(m_{H^{ \pm}}\right)-g_{h H^{+} H^{-}}$plane and $\sin (\beta-\alpha)-f\left(m_{H^{ \pm}}\right)$plane in figure 12 (left) and (right) respectively. Only the regions corresponding to $f\left(m_{H^{ \pm}}\right)>0$ on the righthand side of the vertical line and above the horizontal line are allowed, if one demands $f\left(m_{H^{ \pm}}\right) \geq 80 \mathrm{GeV}$.

\subsubsection{Flipped 2HDM}

Here the up-type quarks and leptons couple to the same doublet while the other doublet couples to down-type quarks. The Yukawa Lagrangian becomes

$$
\mathcal{L}_{\text {Yukawa }}=y_{i j}^{1} \bar{Q}_{i L} \Phi_{1} d_{j R}+y_{i j}^{2} \bar{Q}_{i L} \bar{\Phi}_{2} u_{j R}+y_{i j}^{5} \bar{L}_{i L} \Phi_{2} e_{j R}
$$

which is achieved by imposing $\Phi_{1} \rightarrow-\Phi_{1}$ and $d_{R} \rightarrow-d_{R}$. It is straightforward to show that the scale factors with respect to SM Higgs couplings to fermions and gauge bosons then become:

$$
\begin{aligned}
C_{h t \bar{t}} & =\frac{\cos \alpha}{\sin \beta} \times C_{h t \bar{t}}^{\mathrm{S} M} \\
C_{h b \bar{b}} & =-\frac{\sin \alpha}{\cos \beta} \times C_{h b \bar{b}}^{\mathrm{S} M} \\
C_{h \tau \bar{\tau}} & =\frac{\cos \alpha}{\sin \beta} \times C_{h \tau \bar{\tau}}^{\mathrm{S} M} \\
C_{h V V} & =\sin (\beta-\alpha) \times C_{h V V}^{\mathrm{S} M}
\end{aligned}
$$

and the scaling factors are

$$
\begin{aligned}
\kappa_{t} & =\kappa_{\tau} \\
\kappa_{b} & =\frac{\kappa_{t}^{2}-2 \kappa_{t} \kappa_{v}+1}{\kappa_{t}-\kappa_{v}}
\end{aligned}
$$

Here our 6-dimensional parameter space are comprised of $\kappa_{v}, \kappa_{t}, f_{B B}, f_{W W}, f_{B}, f_{W}$. The $1 \sigma$ and $2 \sigma$ regions are shown in figure 13 (left) in the $\kappa_{v}-\kappa_{t}$ plane and in figure 13 (right) 

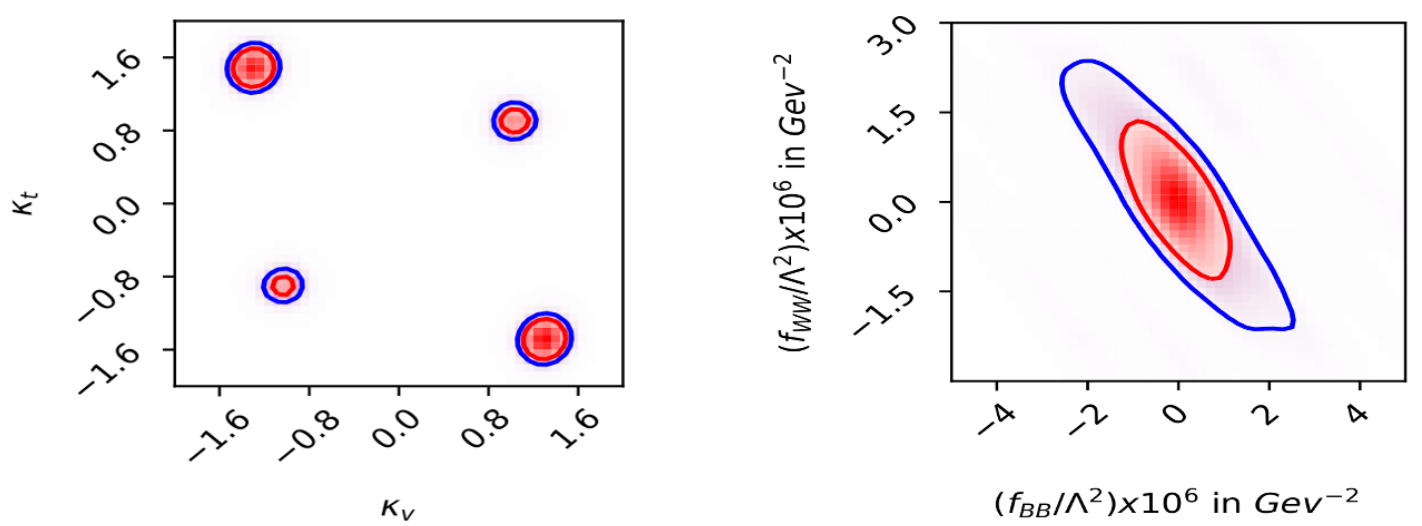

Figure 13. Allowed regions at $1 \sigma(\mathrm{red})$ and $2 \sigma$ (blue) levels in the parameter space of scale factors and dimension-6 couplings in Flipped 2HDM.
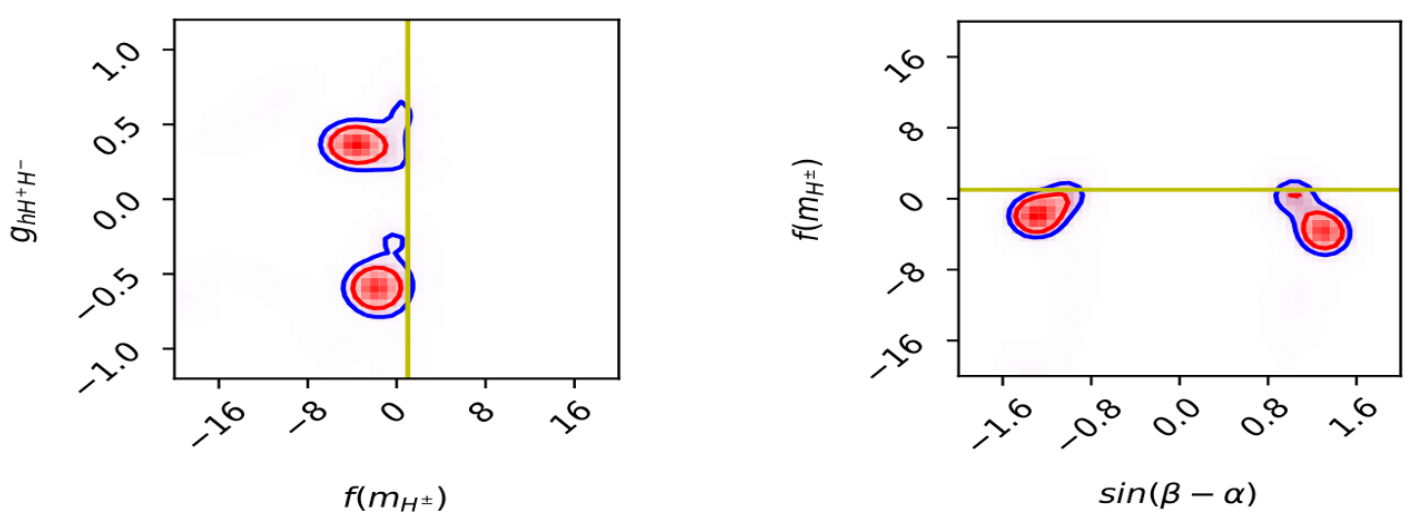

Figure 14. Allowed regions at $1 \sigma(\mathrm{red})$ and $2 \sigma$ (blue) levels in the parameter space of $f\left(m_{H^{ \pm}}\right)-$ $g_{h H^{+} H^{-}}$(left) and $\sin (\beta-\alpha)-f\left(m_{H^{ \pm}}\right)$(right) in Flipped 2HDM.

in $f_{B B^{-}} f_{W W}$ plane, from which one obtains the corresponding regions in the model parameter space, following a strategy similar to the one used previously.

$$
\begin{aligned}
\kappa_{v} & =\sin (\beta-\alpha) \\
\kappa_{t} & =\frac{\cos \alpha}{\sin \beta}=\sin (\beta-\alpha)-\cos (\beta-\alpha) \tan \beta
\end{aligned}
$$

One thus obtains $1 \sigma$ and $2 \sigma$ contours in the $f\left(m_{H^{ \pm}}\right)-g_{h H^{+} H^{-}}$plane and $\sin (\beta-\alpha)$ $f\left(m_{H^{ \pm}}\right)$plane in figure 14 (left) and (right) respectively. Here, too, a lower limit on the charged Higgs mass similar to that for Type-II 2HDM is applicable.

\subsubsection{Comparison among various 2HDMs}

We next ask the question: while allowed regions for the various 2HDM scenarios are identified as above from available data, can some quantities be defined further, which may act as differentiators among them? We are essentially occupied with one such quantity in the rest of this subsection. 

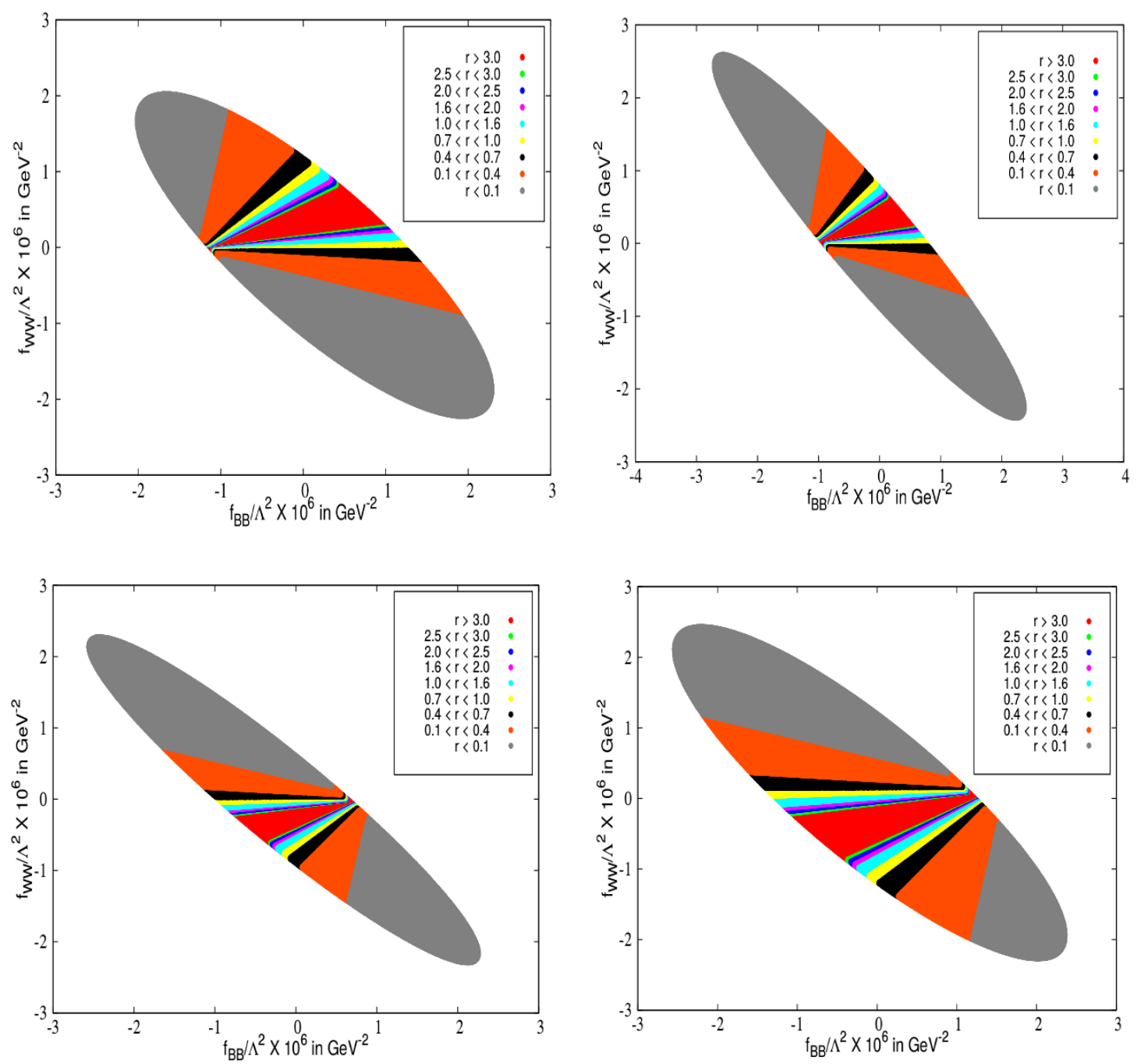

Figure 15. The ratio $r$ in $f_{B B^{-}} f_{W W}$ plane for Type $\mathrm{I}$ (top left), Type II(top right), Leptonspecific(bottom left) and Flipped $2 \mathrm{HDM}$ (bottom right). The ellipses denote the regions allowed at $2 \sigma$ level.

With this in view, we consider the ratio

$$
r=\frac{\mu_{\gamma \gamma}}{\mu_{Z \gamma}}
$$

which essentially reduces to $\frac{\Gamma(h \rightarrow \gamma \gamma)}{\Gamma(h \rightarrow Z \gamma)} / \frac{\Gamma(h \rightarrow \gamma \gamma)_{\mathrm{SM}}}{\Gamma(h \rightarrow Z \gamma)_{\mathrm{SM}}}$, when all the major channels of Higgs production are summed over. We show color-coded regions in the $f_{B B^{-}} f_{W W}$ plane for the four aforesaid types of $2 \mathrm{HDM}$ in figure 15 . As can be seen there, $r$ can range form values below 0.1 to those as large as 3, consistently with the current data. As progressively accumulating data shrink the $2 \sigma$ regions, thereby causing the ellipses to become smaller, the observation of this ratio can narrow down the choice among different models at least partially. One rather striking observation here is the opposite directions of convergence 

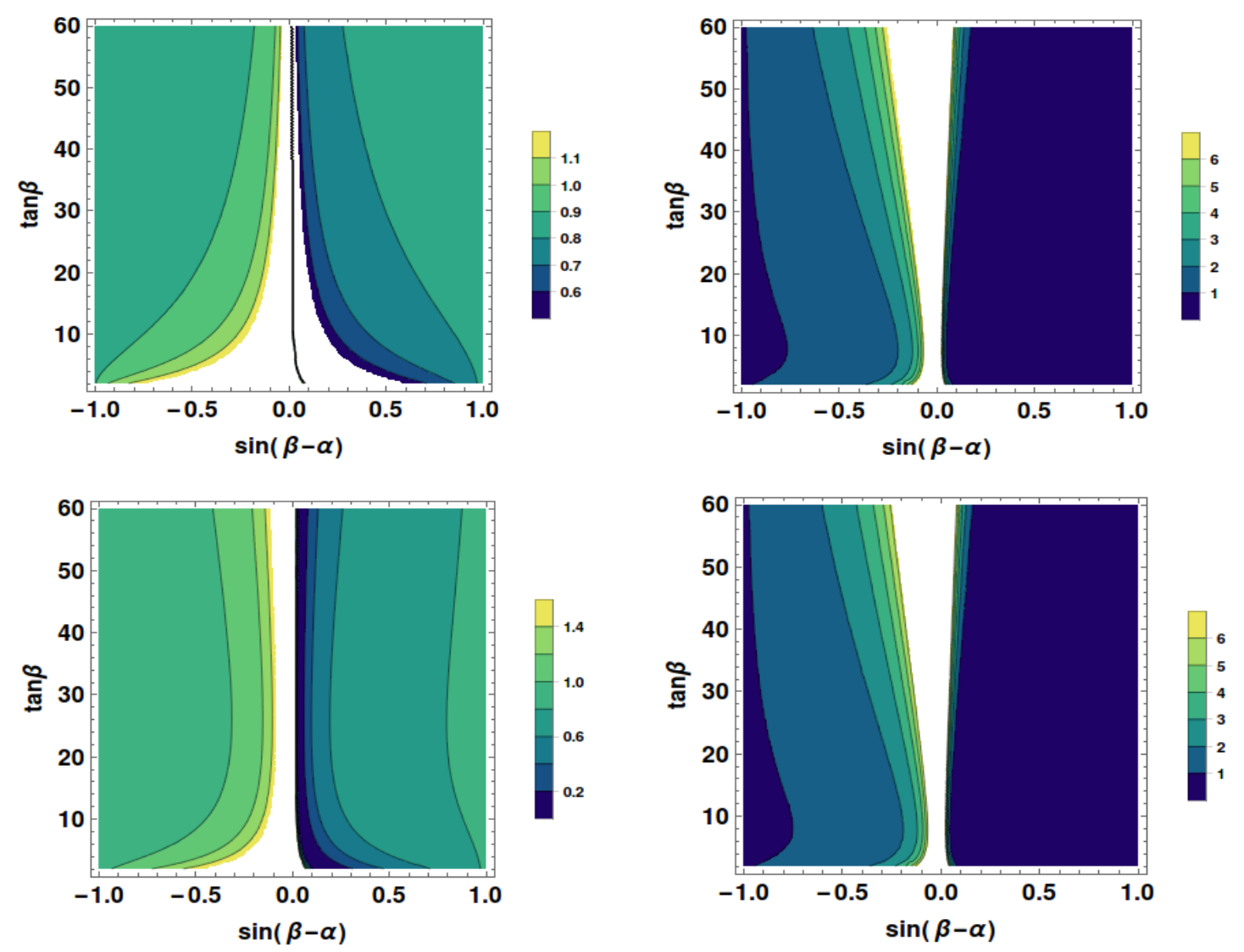

Figure 16. The ratio $r$ in $\sin (\beta-\alpha)$-tan $\beta$ plane for Type I(top left), Type II(top right), Leptonspecific(bottom left) and Flipped $2 \mathrm{HDM}$ (bottom right), where $m_{H^{ \pm}}=600 \mathrm{GeV}$.

of the color-coded regions for Types I and II as against those for the Lepton-specific and Flipped cases. This is a consequence of the fact that the best-fit value for $\kappa_{v}$ is negative for Type I and Type II, whereas it is positive in case of Lepton-specific and Flipped scenarios. The $W$-contribution plays a major role in both $h \rightarrow \gamma \gamma$ and $h \rightarrow Z \gamma$ decays; therefore the sign of the scale factor $\kappa$ of the $W$-loop is the key factor in deciding the pattern of the ratio $r$ via interference with the effective operators. It should also be noted that in general the allowed regions for Type-I and Flipped category are slightly bigger than those of Type-II and Lepton-specific case. While drawing these plots the scale factors $\kappa$ 's have been kept at their best-fit values and the dimension- 6 couplings $f_{B}$ and $f_{W}$ are assumed to be zero.

Color-coded regions corresponding to different ranges of $r$ in the $\sin (\beta-\alpha)$-tan $\beta$ plane are shown in figure 16 . In case of Type I $2 \mathrm{HDM}$, for $\sin (\beta-\alpha) \approx+1$ (the so-called 'alignment limit' [43] indicates $\sin (\beta-\alpha) \approx \pm 1$ ), and low $\tan \beta$, the top loop contribution to $h \rightarrow \gamma \gamma$ increases and interferes destructively with the $W$-loop contribution. The charged Higgs loop also interferes destructively with the $W$ loop. These contributions thus collectively reduce the decay width as compared to its SM value.

On the other hand, in the case of $h \rightarrow Z \gamma$, the top and charged Higgs loops interfere constructively with the $W$-loop. Therefore, despite the marginal fall in the $W$-loop contri- 
bution from the SM value, driven by $\sin ^{2}(\beta-\alpha)$, such constructive interference partially compensates it. Hence for positive $\sin (\beta-\alpha)$ the ratio $r$ is always less than unity.

In the region with $\sin (\beta-\alpha) \approx-1, \Gamma(h \rightarrow \gamma \gamma)$ has a small suppression over the entire chosen range of $\tan \beta$. On the other hand, the top loop contribution to $h \rightarrow Z \gamma$ there decreases more significantly at small $\tan \beta$. Coupled with the effect of constructive interference of the top, $W$ and charged Higgs loops, this reduces the overall $h \rightarrow Z \gamma$ decay width from its SM value. Hence the ratio $r$ in this case can be greater than unity for low $\tan \beta$, as can be seen in figure 16 (top left). The Lepton-specific scenario shows a trend similar to Type I, as is clear from figure 16 (bottom left), since the quark couplings to Higgs are same in both the cases.

Figure 16 shows that, for Type II and Flipped $2 \mathrm{HDM}$, the ratio is always $<1$ if $\sin (\beta-\alpha)$ is not much different from +1 . But in the wrong-sign region where $\sin (\beta-\alpha) \approx-1$ which gives rise to a positive sign in the coupling of Higgs with down-type quarks, $r$ can be greater than 1 for large enough $\tan \beta$. For such $\tan \beta$, the bottom loop contribution to $h \rightarrow \gamma \gamma$ increases. In case of positive $\sin (\beta-\alpha)$ this loop interferes destructively with the $W$ loop and constructively for negative $\sin (\beta-\alpha)$. The top and charged Higgs loops interfere destructively with the $W$ loop in both these cases. Hence the decay width of $h \rightarrow \gamma \gamma$ can be greater than its SM value for negative sign of $\sin (\beta-\alpha)$. For $h \rightarrow Z \gamma$ in the positive $\sin (\beta-\alpha)$ region, the bottom loop constructively interferes with the $W$ and top loop, where in the negative $\sin (\beta-\alpha)$ regime, the bottom loop destructively interferes with the $W$ and top loops. The charged Higgs loop contribution is much smaller compared to the rest of the contributions. At large $\tan \beta$ the contribution increases and therefore the $h \rightarrow Z \gamma$ decay width decreases below its SM value for negative $\sin (\beta-\alpha)$ and increase above that SM value for positive $\sin (\beta-\alpha)$. Thus in general the ratio $r$ can be greater than unity for large $\tan \beta$ and for the wrong sign region for Type II 2HDM, as can be seen from figure 16 (top right). The Flipped 2HDM scenario, too follows the same trend, as is evident from figure 16 (bottom right).

We have checked the validity of the aforementioned analysis over a wide range of charged Higgs masses. In figure 16 we present the results for a charged Higgs mass of $600 \mathrm{GeV}$. But the results remain unaltered even for $m_{H^{ \pm}} \sim \mathcal{O}(\mathrm{TeV})$.

\subsection{Higgs triplet models}

As already mentioned, a pertinent issue for SM has been neutrino masses and mixing. A popular model that explains these, by introducing an extension of the EWSB sector, is the Type-II see-saw mechanism [44-61]. This scenario allows one to dispense with right-handed neutrinos, having instead one or more scalar triplets with hypercharge 2. Rather strong constraints arising from the $\rho$-parameter apply on the vev of the triplet(s), restricting them to values $\mathcal{O} \sim 1 \mathrm{GeV}$. Moreover, a bid to unification via left-right symmetry [62-64] makes use of such triplet scalars.

The bi-large neutrino mixing pattern, the confirmation of the non-vanishing mixing element $\theta_{13}$, and the various suggested mass hierarchies prompt one to set up neutrino mass models which try to connect mass eigenvalues with the mixing angles, thereby adding predictiveness to the theoretical formulation. Such predictiveness is enhanced if the neu- 
trino mass matrix in the flavor basis contains some zero entries, ensured by the imposition of additional symmetries. However, a single triplet fails to make Type II seesaw scenarios consistent with some widely used zero texture models, in particular, those with two-zero texture. On the contrary, such texture can be reproduced if one has two triplets instead of one, thereby suggesting a scenario whose phenomenological implications have been explored in recent works [65] and the collider signatures of this model have been studied in [66]. With this in mind, we analyze below models both with one and two scalar triplet in the light of the recent Higgs data and the contribution of charged scalar loops (including those from doubly charged scalars) embodied in dimension- 6 operators.

\subsubsection{A single-triplet model}

Here a bi-doublet $\Delta$ is added in the Higgs sector. Therefore in addition to the SM Higgs state we have three more Higgs states, namely, doubly charged $\Delta^{++}$, singly charged $\Delta^{+}$ and neutral $\Delta^{0}$ ones.

$$
\Delta=\left(\begin{array}{cc}
\Delta^{+} & \sqrt{2} \Delta^{++} \\
\sqrt{2} \Delta^{0} & -\Delta^{+}
\end{array}\right) .
$$

The vev of the doublet $\phi$ and triplet $\Delta$ can be expressed as

$$
\langle\phi\rangle_{0}=\frac{1}{\sqrt{2}}\left(\begin{array}{l}
0 \\
v
\end{array}\right) \quad \text { and } \quad\langle\Delta\rangle_{0}=\left(\begin{array}{cc}
0 & 0 \\
w & 0
\end{array}\right) .
$$

The scalar potential in its most general form can be written as

$$
\begin{aligned}
V(\phi, \Delta)= & a \phi^{\dagger} \phi+\frac{b}{2} \operatorname{Tr}\left(\Delta^{\dagger} \Delta\right)+\left(\phi^{\dagger} \phi\right)^{2}+\frac{d}{4}\left(\operatorname{Tr}\left(\Delta^{\dagger} \Delta\right)\right)^{2} \\
& +\frac{e-h}{2} \phi^{\dagger} \phi \operatorname{Tr}\left(\Delta^{\dagger} \Delta\right)+\frac{f}{4} \operatorname{Tr}\left(\Delta^{\dagger} \Delta^{\dagger}\right) \operatorname{Tr}(\Delta \Delta) \\
& +h \phi^{\dagger} \Delta^{\dagger} \Delta \phi+\left(t \phi^{\dagger} \Delta \tilde{\phi}+\text { H.c. }\right)
\end{aligned}
$$

where $\tilde{\phi} \equiv i \tau_{2} \phi^{*}$.

We neglect CP-violation for simplicity. Thus all the coefficients in the scalar potential are assumed to be real. To ensure that EWSB is driven predominantly by the doublet state, certain restrictions are imposed on the parameters. The choice $a<0, b>0$, takes care of this. In addition, $w \ll v$, as required by the $\rho$-parameter constraint. The doublettriplet mixing should be small in general. In addition, we have confined ourselves to cases where all quartic couplings are perturbative. These requirements guide us to choose the parameter space in the following manner:

$$
a, b \sim v^{2} ; \quad c, d, e, f, h \sim 1 ; \quad|t| \ll v .
$$

After spontaneous symmetry breaking, we are left with five physical states, namely, two neutral scalars (one SM-like among them and one heavier), one each of singly-and doubly-charged scalars, one pseudoscalar. $\mathrm{SU}(2)$ invariance of the Lagrangian plus the smallness demands of doublet-triplet mixing force masses of the triplet-dominated states 

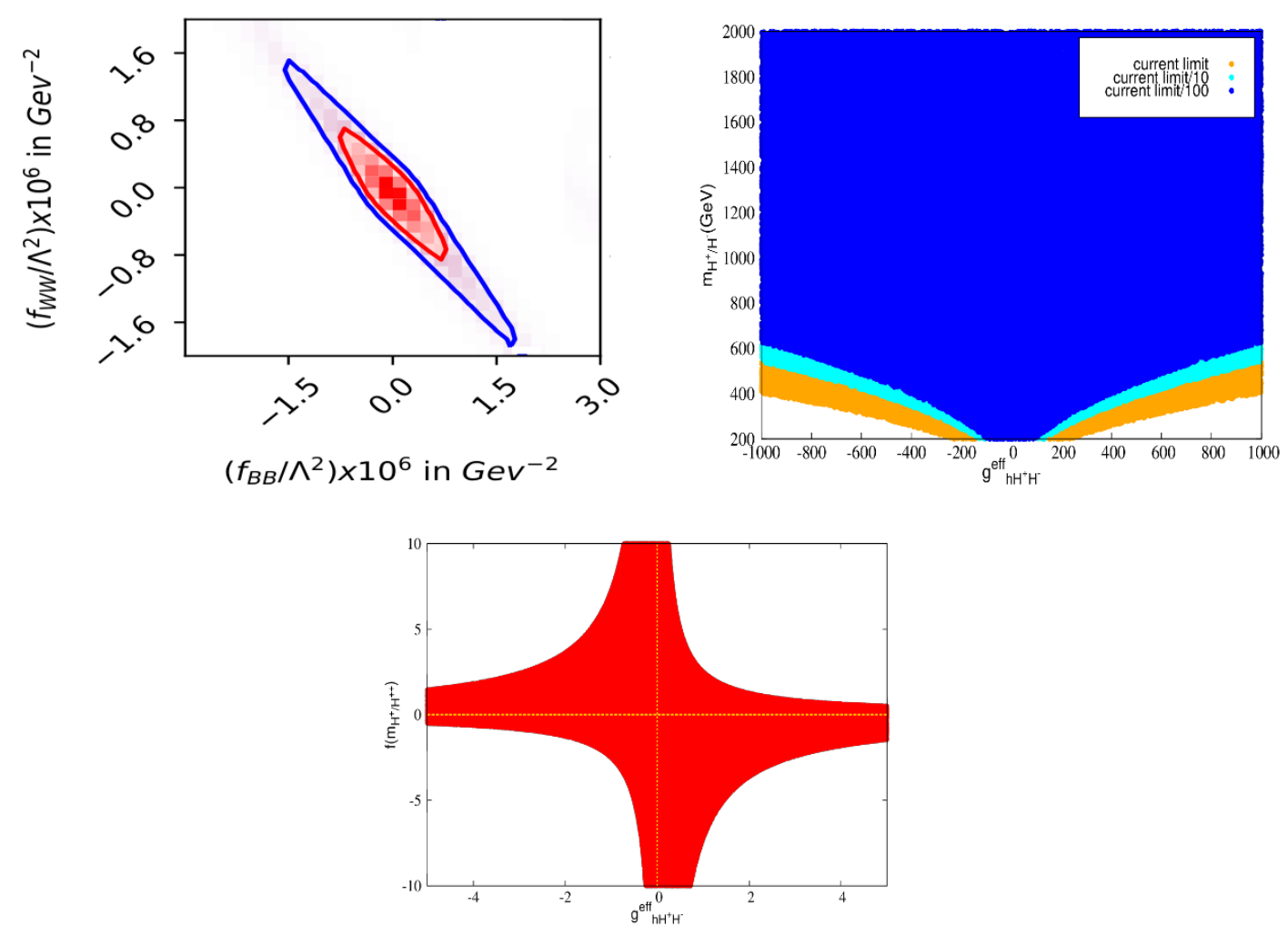

Figure 17. Allowed regions at $1 \sigma$ (red) and $2 \sigma$ (blue) levels in the parameter space of scale factors and dimension- 6 couplings in the triplet scenario.

to be nearly degenerate. In such a situation SM-like Higgs couplings to fermions and gauge bosons are practically unaltered. Therefore we can assume

$$
\kappa_{v} \approx \kappa_{t} \approx \kappa_{b} \approx \kappa_{\tau} \approx 1
$$

Both the charged Higgs states will contribute to the loop-induced decays of the SM-like Higgs $h$, the contribution of the doubly charged Higgs loop to the width being four times that of the singly charged one. The decay rates of $h \rightarrow \gamma \gamma$ and $h \rightarrow Z \gamma$ in single-triplet case are given by [33-36]. We also discuss them in detail in appendix B.

Since the couplings of $h$ to fermions and gauge bosons are practically unchanged, the one-loop contribution to $h \rightarrow \gamma \gamma, Z \gamma$ via the top-and $\mathrm{W}$-loops are same as in SM. The loops induced by the singly-and doubly-charged scalars can, as before, be directly related to the dimension- 6 couplings.

The $1 \sigma$ and $2 \sigma$ regions in the $f_{B B^{-}} f_{W W}$ plane can now be obtained on the assumption that all $\kappa$ 's are unity. Based on this, the $1 \sigma$ and $2 \sigma$ contours in the $f_{B B^{-}} f_{W W}$ plane are found in figure 17 (top left). These are then directly translated into limits on the mass of the degenerate heavy scalar bosons.

One can define an effective coupling as $g_{h H^{+} H^{-}}^{\text {eff }}=\tilde{g}_{h H^{+} H^{-}}+4 \tilde{g}_{h H^{++} H^{--}}$, which can be directly extracted from equation (B.1) to be found in the appendix B. Figure 17 (top right) 
is the translation of the $2 \sigma$ region of figure 17 (top left) into the parameter space spanned by the triplet mass scale $m_{H^{ \pm} / H^{ \pm \pm}}$and the effective coupling $g_{h H^{+} H^{-}}^{\text {eff }}$. The yellow region (over and above the cyan and blue region) here is allowed by the current limit on $f_{B B}$ and $f_{W W}$. The region in cyan (over and above the blue region) will be allowed if the limit on $f_{B B}$ and $f_{W W}$ improves by a factor of 10 . The blue region represents the corresponding projection assuming 100-fold improvement of the same limits. Figure 17 (bottom centre) maps the same limit to the $f\left(m_{H^{ \pm} / H^{ \pm \pm}}\right)-g_{h H^{+} H^{-}}^{\text {eff }}$ parameter space, showing directly the allowed values of the resultant loop function in this scenario.

\subsubsection{A model with two triplets}

Based on the motivations discussed above, we next take up a scenario with two triplet scalars with $Y=2$, namely, $\Delta_{1}, \Delta_{2}$, each of which can be expressed as a bi-doublet:

$$
\Delta_{1}=\left(\begin{array}{cc}
\delta_{1}^{+} & \sqrt{2} \delta_{1}^{++} \\
\sqrt{2} \delta_{1}^{0} & -\delta_{1}^{+}
\end{array}\right) \quad \text { and } \quad \Delta_{2}=\left(\begin{array}{cc}
\delta_{2}^{+} & \sqrt{2} \delta_{2}^{++} \\
\sqrt{2} \delta_{2}^{0} & -\delta_{2}^{+}
\end{array}\right)
$$

The vevs of the scalar triplets are given by

$$
\left\langle\Delta_{1}\right\rangle_{0}=\left(\begin{array}{cc}
0 & 0 \\
w_{1} & 0
\end{array}\right) \quad \text { and } \quad\left\langle\Delta_{2}\right\rangle_{0}=\left(\begin{array}{cc}
0 & 0 \\
w_{2} & 0
\end{array}\right) .
$$

The vev of the Higgs doublet is given by eq. (4.35).

The scalar potential in this model involving $\phi, \Delta_{1}$ and $\Delta_{2}$ can be written as

$$
\begin{aligned}
V\left(\phi, \Delta_{1}, \Delta_{2}\right)= & a \phi^{\dagger} \phi+\frac{1}{2} b_{k l} \operatorname{Tr}\left(\Delta_{k}^{\dagger} \Delta_{l}\right)+c\left(\phi^{\dagger} \phi\right)^{2}+\frac{1}{4} d_{k l}\left(\operatorname{Tr}\left(\Delta_{k}^{\dagger} \Delta_{l}\right)\right)^{2} \\
& +\frac{1}{2}\left(e_{k l}-h_{k l}\right) \phi^{\dagger} \phi \operatorname{Tr}\left(\Delta_{k}^{\dagger} \Delta_{l}\right)+\frac{1}{4} f_{k l} \operatorname{Tr}\left(\Delta_{k}^{\dagger} \Delta_{l}^{\dagger}\right) \operatorname{Tr}\left(\Delta_{k} \Delta_{l}\right) \\
& +h_{k l} \phi^{\dagger} \Delta_{k}^{\dagger} \Delta_{l} \phi+g \operatorname{Tr}\left(\Delta_{1}^{\dagger} \Delta_{2}\right) \operatorname{Tr}\left(\Delta_{2}^{\dagger} \Delta_{1}\right)+g^{\prime} \operatorname{Tr}\left(\Delta_{1}^{\dagger} \Delta_{1}\right) \operatorname{Tr}\left(\Delta_{2}^{\dagger} \Delta_{2}\right) \\
& +\left(t_{k} \phi^{\dagger} \Delta_{k} \tilde{\phi}+\text { H.c. }\right) .
\end{aligned}
$$

The assumption that Higgs sector is CP-conserving prompts us to take all the parameters in equation (4.41) as real. The parameters lie in ranges very similar to those in the single-triplet case. Here, too, the vev of the two triplets are kept on the order of a GeV. This ensures negligible mixing between the doublet and the triplets, again implying

$$
\kappa_{v} \approx \kappa_{t} \approx \kappa_{b} \approx \kappa_{\tau} \approx 1
$$

The decay rates for $h \rightarrow \gamma \gamma$ and $h \rightarrow Z \gamma$ in the two-triplet case are given by [65]. We have also discussed them in appendix B.

The allowed regions in the parameter space are illustrated in figures 18 and 19, in the limit where the mixing between the two triplets is negligible. ${ }^{4}$ The following relations then hold:

$$
m_{H_{1}^{ \pm}} \approx m_{H_{1}^{ \pm \pm}}, \quad m_{H_{2}^{ \pm}} \approx m_{H_{2}^{ \pm \pm}}
$$

\footnotetext{
${ }^{4}$ This assumption in general has no justification; and is made here to enable us to express the singlyand doubly-charged scalar loop functions to have the same value in each triplet. If there is substantial mixing between them, one will have fours mass eigenstates altogether, and the loop functions will assume their values accordingly.
} 


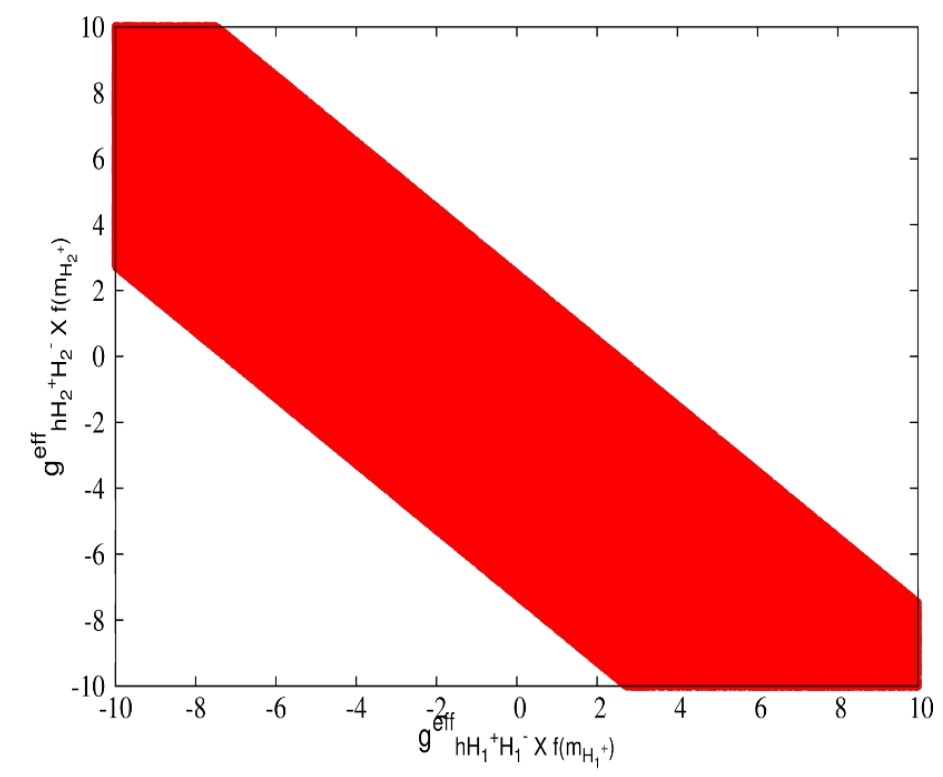

Figure 18. The allowed region in the plane spanned by $g_{h H_{1}^{+} H_{1}^{-}}^{\mathrm{eff}} \times f\left(m_{H_{1}^{ \pm}}\right)$and $g_{h H_{2}^{+} H_{2}^{-}}^{\mathrm{eff}} \times f\left(m_{H_{2}^{ \pm}}\right)$ at $2 \sigma$ level.

and again we define effective couplings as in the singlet case:

$$
\begin{aligned}
& g_{h H_{1}^{+} H_{1}^{-}}^{\text {eff }}=\tilde{g}_{1_{h H_{1}^{+} H_{1}^{-}}}+4 \tilde{g}_{1} h H_{1}^{++} H_{1}^{--} \\
& g_{h H_{2}^{+} H_{2}^{-}}^{\text {eff }}=\tilde{g}_{2} h H_{2}^{+} H_{2}^{-}+4 \tilde{g}_{2} h H_{2}^{++} H_{2}^{--}
\end{aligned}
$$

In figure 18, we plot the allowed region in the $g_{h H_{1}^{+} H_{1}^{-}}^{\text {eff }} \times f\left(m_{H_{1}^{ \pm}}\right)-g_{h H_{2}^{+} H_{2}^{-}}^{\text {eff }} \times f\left(m_{H_{2}^{ \pm}}\right)$ plane at the $2 \sigma$ level, following the same procedure as in the previous cases. In figure 19 we present the color-coded diagram of the ratio $r$ (as defined earlier) in the $f_{B B^{-}} f_{W W}$ plane for the triplet case. In this case the dimension- 6 couplings $f_{B}$ and $f_{W}$ are assumed to be zero. If one compares this plot with figure 15 , one can see that the region corresponding to $r<0.1$ is smaller in case of triplet models than for any kind of 2HDM.

\section{Conclusion}

We have explored the scope of new physics in the Higgs sector in the light of the modified couplings with multiplicative scale factors $\kappa_{i}$ as well as loop contributions parametrized by effective dimension- 6 operators $\left(\mathcal{O}_{B B}, \mathcal{O}_{W W}, \mathcal{O}_{B}\right.$ and $\left.\mathcal{O}_{W}\right)$. For the loop-induced decays $h \rightarrow \gamma \gamma, Z \gamma$, the SM-like loops are merely modified by the appropriate $\kappa$-parameters. In addition charged scalars may contribute in the loop induced processes such as $h \rightarrow \gamma \gamma, Z \gamma$. Thus these loop amplitudes contain effect of both the scale factors and the coefficients of the dimension- 6 operators. We have imposed all existing experimental constraints and 


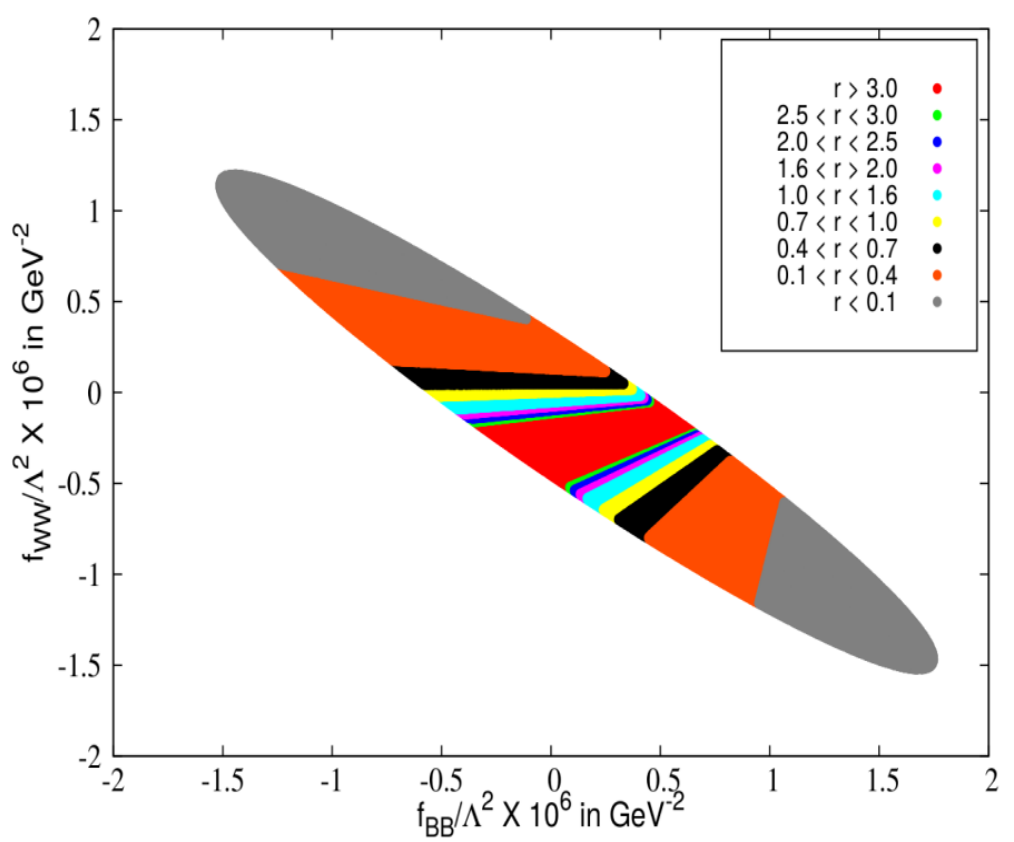

Figure 19. The ratio $r$ in $f_{B B^{-}} f_{W W}$ plane for triplet case. The ellipse denotes the region allowed at $2 \sigma$ level.

found the regions of parameter space, which are allowed at the 1 and $2 \sigma$ levels. While doing so we have taken into account the non-trivial correlation that may exist between various production mechanisms. Although there is no observation of $h \rightarrow Z \gamma$, the upper limit on this channel from current data puts considerable constraints on the parameter space spanned by $f_{B B}, f_{W W}, f_{B}$ and $f_{W}$.

We have tried next to establish connections between this model-independent effective field theory approach and specific new physics models with extended Higgs sector, namely various types of $2 \mathrm{HDM}$ and Higgs triplet models. We have observed that even when the heavy Higgs states in question are not much higher than the EWSB scale, it is possible to describe the new physics in terms of gauge-invariant effective operators. Thanks to this observation, we have made a correspondence between the model-independent and modeldependent approach and translated the limit obtained on the effective operators into those on specific model parameters. Thereafter, we have used the ratio between signal strengths in the $h \rightarrow \gamma \gamma$ and $h \rightarrow Z \gamma$ channel to show that this ratio helps in disentangling the effect of various types of new physics.

\section{A Decay amplitudes in $2 \mathrm{HDM}$}

The one-loop decay widths for $h \rightarrow \gamma \gamma$ and $h \rightarrow Z \gamma$ under the framework of 2HDM can be written as:

$$
\Gamma(h \rightarrow \gamma \gamma)=\frac{\alpha^{2} g^{2}}{1024 \pi^{3}} \frac{m_{h}^{3}}{m_{W}^{2}}\left|\sum_{i} N_{c i} R_{i}^{h} e_{i}^{2} F_{i}\right|^{2}
$$


Where $i$ denotes spin index corresponding to spin-0(charged Higgs), spin-1/2(top and bottom), and spin-1 $(W), e_{i}$ is the electric charge in units of $e$.

$$
\begin{aligned}
F_{W} & =2+3 \tau+3 \tau(2-\tau) f^{0}(\tau) \\
F_{t / b} & =-2 \tau\left[1+(1-\tau) f^{0}(\tau)\right] \\
F_{H^{ \pm}} & =\tau\left[1-\tau f^{0}(\tau)\right]
\end{aligned}
$$

and

$$
\tau=\frac{4 m_{i}^{2}}{m_{h}^{2}}
$$

where $m_{i}$ is the mass of the particle running in the loop.

$$
\begin{aligned}
f^{0}(\tau) & =\left(\begin{array}{ll}
{\left[\sin ^{-1}(\sqrt{1 / \tau})\right]^{2},} & \text { if } \tau \geq 1, \\
-\frac{1}{4}\left[\ln \left(\eta_{+} / \eta_{-}\right)-i \pi\right]^{2}, & \text { if } \tau<1
\end{array}\right) \\
\Gamma(h \rightarrow Z \gamma) & =\frac{\alpha^{2} g^{2}}{512 \pi^{3}} \frac{m_{h}^{3}}{m_{W}^{2}}\left(1-\frac{m_{Z}^{2}}{m_{h}^{2}}\right)^{3}\left|\sum_{i} N_{c i} R_{i}^{h} A_{i}\right|^{2}
\end{aligned}
$$

The index i carries the same meaning as mentioned previously.

$$
\begin{aligned}
A_{W} & =-\cot \theta_{W}\left(4\left(3-\tan ^{2} \theta_{W}\right) I_{2}\left(\tau_{W}, \lambda_{W}\right)+\left[\left(1+\frac{2}{\tau_{W}}\right) \tan ^{2} \theta_{W}-\left(5+\frac{2}{\tau_{W}}\right)\right] I_{1}\left(\tau_{W}, \lambda_{W}\right)\right) \\
A_{f} & =\frac{-2 e_{f}\left(T_{f}^{3 L}-2 e_{f} \sin ^{2} \theta_{W}\right)}{\sin \theta_{W} \cos \theta_{W}}\left[I_{1}\left(\tau_{f}, \lambda_{f}\right)-I_{2}\left(\tau_{f}, \lambda_{f}\right)\right],
\end{aligned}
$$

where $f$ stands for $t$ or $b$ quark

$$
A_{H^{ \pm}}=\frac{1-2 \sin ^{2} \theta_{W}}{\cos \theta_{W} \sin \theta_{W}} I_{1}\left(\tau_{H^{ \pm}}, \lambda_{H^{ \pm}}\right) \frac{m_{W}^{2}}{m_{H^{ \pm}}^{2}}
$$

and

$$
\tau_{i}=\frac{4 m_{i}^{2}}{m_{h}^{2}}, \quad \lambda_{i}=\frac{4 m_{i}^{2}}{m_{Z}^{2}}
$$

The functions $I_{1}$ and $I_{2}$ are defined as

$$
\begin{aligned}
I_{1}(a, b) & =\frac{a b}{2(a-b)}+\frac{a^{2} b^{2}}{2(a-b)^{2}}\left[f^{0}(a)-f^{0}(b)\right]+\frac{a^{2} b}{(a-b)^{2}}[g(a)-g(b)], \\
I_{2}(a, b) & =-\frac{a b}{2(a-b)}\left[f^{0}(a)-f^{0}(b)\right] \\
g(\tau) & =\left(\begin{array}{cc}
\sqrt{\tau-1} \sin ^{-1}(\sqrt{1 / \tau)}, & \text { if } \tau \geq 1, \\
-\frac{1}{2} \sqrt{1-\tau}\left[\ln \left(\eta_{+} / \eta_{-}\right)-i \pi\right], & \text { if } \tau<1
\end{array}\right)
\end{aligned}
$$

$R_{i}^{h}$ in equations (A.1) and (A.5) denotes the scale factor for $W$, top and bottom loop compared to their SM values. Essentially, $R_{W}^{h}$ and $R_{f}^{h}$ are equivalent to the scaling of $h V V$ and $h f \bar{f}$ coupling with respect to their SM predictions. $R_{H^{ \pm}}^{h}$ is the trilinear Higgs coupling $g_{h H^{+} H^{-}}$in all two-Higgs doublet models. The scale factors $R_{W}^{h}, R_{f}^{h}$ and $R_{H^{ \pm}}^{h}$ can be expressed in terms of the model parameters, namely, $\tan \beta$ and $\sin (\beta-\alpha)$. The Higgs potential is general and is valid for all types of $2 \mathrm{HDM}$. 


\section{B Decay amplitudes in models with triplet scalar(s)}

\section{B.1 Single triplet case}

The decay amplitude for $h \rightarrow \gamma \gamma$ in models with single triplet is given by

$$
\begin{aligned}
\Gamma(h \rightarrow \gamma \gamma)= & \frac{\alpha^{2} G_{F} m_{h}^{3}}{128 \sqrt{2} \pi^{3}} \mid \sum_{f} N_{c} Q_{f}^{2} g_{h f \bar{f}} A_{1 / 2}^{\gamma \gamma}\left(\tau_{h}^{f}\right)+g_{h W^{+} W^{-}} A_{1}^{\gamma \gamma}\left(\tau_{h}^{W}\right) \\
& +\tilde{g}_{h H^{ \pm} H^{\mp}} A_{0}^{\gamma \gamma}\left(\tau_{h}^{H^{ \pm}}\right)+\left.4 \tilde{g}_{h H^{ \pm \pm} H^{\mp \mp}} A_{0}^{\gamma \gamma}\left(\tau_{h}^{H^{ \pm \pm}}\right)\right|^{2}
\end{aligned}
$$

where $\alpha$ is the fine-structure constant, $G_{F}$ is the Fermi coupling constant, $N_{c}=3(1)$ for quarks (leptons), and $Q_{f}$ is the electric charge of the fermion in the loop.

The decay rate for $h \rightarrow Z \gamma$ in single-triplet case given by

$$
\begin{aligned}
& \Gamma(h \rightarrow Z \gamma)=\frac{\alpha^{2} m_{h}^{3}}{128 \pi^{3} v^{2}}\left(1-\frac{m_{Z}^{2}}{m_{h}^{2}}\right)^{3} \mid \frac{1}{\sin \theta_{W} \cos \theta_{W}} \sum_{f} N_{c} Q_{f}\left(2 I_{3}^{f}-4 Q_{f} \sin ^{2} \theta_{W}\right) A_{1 / 2}^{Z \gamma}\left(\tau_{h}^{f}, \tau_{Z}^{f}\right) \\
& +\cot \theta_{W} g_{h W^{+} W^{-}} A_{1}^{Z \gamma}\left(\tau_{h}^{W}, \tau_{Z}^{W}\right)-2 g_{Z H^{ \pm} H^{\mp}} \tilde{g}_{h H^{ \pm} H^{\mp}} A_{0}^{Z \gamma}\left(\tau_{h}^{H^{ \pm}}, \tau_{Z}^{H^{ \pm}}\right) \\
& -\left.4 g_{Z H^{ \pm \pm} H_{\mp \mp}} \tilde{g}_{h H^{ \pm \pm} H \mp \mp} A_{0}^{Z \gamma}\left(\tau_{h}^{H^{ \pm \pm}}, \tau_{Z}^{H^{ \pm \pm}}\right)\right|^{2},
\end{aligned}
$$

where $\tau_{h}^{i}=4 m_{i}^{2} / m_{h}^{2}, \tau_{Z}^{i}=4 m_{i}^{2} / m_{Z}^{2}\left(i=f, W, H^{ \pm}, H^{ \pm \pm}\right)$, and $I_{3}^{f}$ are the third component of isospin of the fermion.

$$
\begin{aligned}
g_{Z H^{ \pm} H^{\mp}} & =-\tan \theta_{W}, \\
g_{Z H^{ \pm \pm} H^{\mp \mp}} & =2 \cot 2 \theta_{W}
\end{aligned}
$$

The relevant loop functions are

$$
\begin{aligned}
A_{0}^{\gamma \gamma}(x) & =-x^{2}\left[x^{-1}-f\left(x^{-1}\right)\right], \\
A_{1 / 2}^{\gamma \gamma}(x) & =2 x^{2}\left[x^{-1}+\left(x^{-1}-1\right) f\left(x^{-1}\right)\right], \\
A_{1}^{\gamma \gamma}(x) & =-x^{2}\left[2 x^{-2}+3 x^{-1}+3\left(2 x^{-1}-1\right) f\left(x^{-1}\right)\right], \\
A_{0}^{Z \gamma}(x, y) & =I_{1}(x, y), \\
A_{1 / 2}^{Z \gamma}(x, y) & =I_{1}(x, y)-I_{2}(x, y), \\
A_{1}^{Z \gamma}(x, y) & =4\left(3-\tan ^{2} \theta_{W}\right) I_{2}(x, y)+\left[\left(1+2 x^{-1}\right) \tan ^{2} \theta_{W}-\left(5+2 x^{-1}\right)\right] I_{1}(x, y)
\end{aligned}
$$

where

$$
\begin{aligned}
& I_{1}(x, y)=\frac{x y}{2(x-y)}+\frac{x^{2} y^{2}}{2(x-y)^{2}}\left[f\left(x^{-1}\right)-f\left(y^{-1}\right)\right]+\frac{x^{2} y}{(x-y)^{2}}\left[g\left(x^{-1}\right)-g\left(y^{-1}\right)\right], \\
& I_{2}(x, y)=-\frac{x y}{2(x-y)}\left[f\left(x^{-1}\right)-f\left(y^{-1}\right)\right],
\end{aligned}
$$

with the functions $f(x)$ and $g(x)$ in the range $x<1$, given by

$$
f(x)=\left(\sin ^{-1} \sqrt{x}\right)^{2}, \quad g(x)=\sqrt{x^{-1}-1}\left(\sin ^{-1} \sqrt{x}\right) .
$$




\section{B.2 Two-triplet case}

The decay width for the process $h \rightarrow \gamma \gamma$ in the two-triplet case is given as

$$
\begin{aligned}
\Gamma(h \rightarrow \gamma \gamma)= & \frac{\alpha^{2} G_{F} m_{h}^{3}}{128 \sqrt{2} \pi^{3}} \mid \sum_{f} N_{c} Q_{f}^{2} g_{h f f} A_{1 / 2}^{\gamma \gamma}\left(\tau_{h}^{f}\right)+g_{h W^{+} W^{-}} A_{1}^{\gamma \gamma}\left(\tau_{h}^{W}\right) \\
& +\left.\sum_{i=1,2}\left(\tilde{g}_{i h H_{i}^{ \pm} H_{i}^{\mp}} A_{0}^{\gamma \gamma}\left(\tau_{h}^{H_{i}^{ \pm}}\right)+4 \tilde{g}_{i h H_{i}^{ \pm \pm} H_{i}^{\mp \mp}} A_{0}^{\gamma \gamma}\left(\tau_{h}^{H_{i}^{ \pm \pm}}\right)\right)\right|^{2} .
\end{aligned}
$$

The decay rate for $h \rightarrow Z \gamma$ in the two-triplet case is given by

$$
\begin{aligned}
& \Gamma(h \rightarrow Z \gamma)=\frac{\alpha^{2} m_{h}^{3}}{128 \pi^{3} v^{2}}\left(1-\frac{m_{Z}^{2}}{m_{h}^{2}}\right)^{3} \mid \frac{1}{\sin \theta_{W} \cos \theta_{W}} \sum_{f} N_{c} Q_{f}\left(2 I_{3}^{f}-4 Q_{f} \sin ^{2} \theta_{W}\right) A_{1 / 2}^{Z \gamma}\left(\tau_{h}^{f}, \tau_{Z}^{f}\right) \\
& +\cot \theta_{W} g_{h W^{+} W^{-}} A_{1}^{Z \gamma}\left(\tau_{h}^{W}, \tau_{Z}^{W}\right)-\sum_{i=1,2}\left(2 g_{Z H_{i}^{ \pm} H_{i}^{\mp}} \tilde{g}_{i h H_{i}^{ \pm} H_{i}^{\mp}} A_{0}^{Z \gamma}\left(\tau_{h}^{H_{i}^{ \pm}}, \tau_{Z}^{H_{i}^{ \pm}}\right)\right.
\end{aligned}
$$

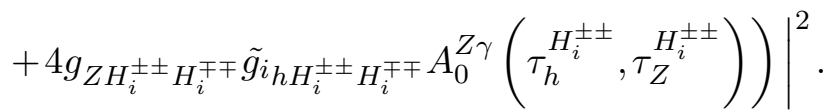

All the quantities in equation (B.8) and (B.9) are given in equations (B.3)-(B.7).

\section{Acknowledgments}

We thank Satyaki Bhattacharya and Arpan Kar for useful discussions at various stages of the work. This work was supported by funding available from the Department of Atomic Energy, Government of India, for the Regional Centre for Accelerator-based Particle Physics (RECAPP), Harish-Chandra Research Institute. The authors acknowledge the hospitality of Indian Association for the Cultivation of Science, Kolkata, where a part of the study was carried out.

Open Access. This article is distributed under the terms of the Creative Commons Attribution License (CC-BY 4.0), which permits any use, distribution and reproduction in any medium, provided the original author(s) and source are credited.

\section{References}

[1] ATLAS and CMS collaborations, Measurements of the Higgs boson production and decay rates and constraints on its couplings from a combined ATLAS and CMS analysis of the LHC pp collision data at $\sqrt{s}=7$ and 8 TeV, JHEP 08 (2016) 045 [arXiv:1606.02266] [INSPIRE].

[2] S. Banerjee, S. Mukhopadhyay and B. Mukhopadhyaya, New Higgs interactions and recent data from the LHC and the Tevatron, JHEP 10 (2012) 062 [arXiv:1207.3588] [INSPIRE].

[3] E. Massó and V. Sanz, Limits on anomalous couplings of the Higgs boson to electroweak gauge bosons from LEP and the LHC, Phys. Rev. D 87 (2013) 033001 [arXiv:1211.1320] [INSPIRE].

[4] T. Corbett, O.J.P. Eboli, J. Gonzalez-Fraile and M.C. Gonzalez-Garcia, Robust Determination of the Higgs Couplings: Power to the Data, Phys. Rev. D 87 (2013) 015022 [arXiv:1211.4580] [INSPIRE]. 
[5] A. Falkowski, F. Riva and A. Urbano, Higgs at last, JHEP 11 (2013) 111 [arXiv:1303.1812] [INSPIRE].

[6] T. Corbett, O.J.P. É boli, J. Gonzalez-Fraile and M.C. Gonzalez-Garcia, Determining Triple Gauge Boson Couplings from Higgs Data, Phys. Rev. Lett. 111 (2013) 011801 [arXiv: 1304.1151] [INSPIRE].

[7] B. Dumont, S. Fichet and G. von Gersdorff, A Bayesian view of the Higgs sector with higher dimensional operators, JHEP 07 (2013) 065 [arXiv: 1304.3369] [INSPIRE].

[8] J.S. Gainer, J. Lykken, K.T. Matchev, S. Mrenna and M. Park, Geolocating the Higgs Boson Candidate at the LHC, Phys. Rev. Lett. 111 (2013) 041801 [arXiv:1304.4936] [INSPIRE].

[9] T. Corbett, O.J.P. É boli, J. Gonzalez-Fraile and M.C. Gonzalez-Garcia, Robust determination of the scalar boson couplings, in Proceedings, 48th Rencontres de Moriond on Electroweak Interactions and Unified Theories: La Thuile, Italy, March 2-9, 2013, pp. 257-264, arXiv:1306.0006 [INSPIRE].

[10] J. Elias-Miro, J.R. Espinosa, E. Massó and A. Pomarol, Higgs windows to new physics through $d=6$ operators: constraints and one-loop anomalous dimensions, JHEP 11 (2013) 066 [arXiv: 1308.1879] [INSPIRE].

[11] M.B. Einhorn and J. Wudka, Higgs-Boson Couplings Beyond the Standard Model, Nucl. Phys. B 877 (2013) 792 [arXiv: 1308.2255] [inSPIRE].

[12] A. Pomarol and F. Riva, Towards the Ultimate SM Fit to Close in on Higgs Physics, JHEP 01 (2014) 151 [arXiv: 1308. 2803] [INSPIRE].

[13] S. Banerjee, S. Mukhopadhyay and B. Mukhopadhyaya, Higher dimensional operators and the LHC Higgs data: The role of modified kinematics, Phys. Rev. D 89 (2014) 053010 [arXiv: 1308.4860] [INSPIRE].

[14] J. de Blas, O. Eberhardt and C. Krause, Current and Future Constraints on Higgs Couplings in the Nonlinear Effective Theory, JHEP 07 (2018) 048 [arXiv:1803.00939] [INSPIRE].

[15] S. Jana and S. Nandi, New Physics Scale from Higgs Observables with Effective Dimension-6 Operators, Phys. Lett. B 783 (2018) 51 [arXiv:1710.00619] [inSPIRE].

[16] S. Dwivedi, D.K. Ghosh, B. Mukhopadhyaya and A. Shivaji, Constraints on CP-violating gauge-Higgs operators, Phys. Rev. D 92 (2015) 095015 [arXiv:1505.05844] [INSPIRE].

[17] J.F. Gunion, H.E. Haber, G.L. Kane and S. Dawson, The Higgs Hunter's Guide, Front. Phys. 80 (2000) 1 [INSPIRE].

[18] K. Hagiwara, S. Ishihara, R. Szalapski and D. Zeppenfeld, Low-energy constraints on electroweak three gauge boson couplings, Phys. Lett. B 283 (1992) 353 [INSPIRE].

[19] K. Hagiwara, S. Ishihara, R. Szalapski and D. Zeppenfeld, Low-energy effects of new interactions in the electroweak boson sector, Phys. Rev. D 48 (1993) 2182 [INSPIRE].

[20] CMS collaboration, Measurements of properties of the Higgs boson in the diphoton decay channel with the full 2016 data set, CMS-PAS-HIG-16-040 (2016).

[21] ATLAS collaboration, Measurements of Higgs boson properties in the diphoton decay channel with $36.1 \mathrm{fb}^{-1} \mathrm{pp}$ collision data at the center-of-mass energy of $13 \mathrm{TeV}$ with the ATLAS detector, ATLAS-CONF-2017-045 (2017).

[22] ATLAS collaboration, Searches for the $Z \gamma$ decay mode of the Higgs boson and for new high-mass resonances in pp collisions at $\sqrt{s}=13$ TeV with the ATLAS detector, JHEP 10 (2017) 112 [arXiv:1708.00212] [INSPIRE]. 
[23] S. Banerjee, T. Mandal, B. Mellado and B. Mukhopadhyaya, Cornering dimension-6 HVV interactions at high luminosity LHC: the role of event ratios, JHEP 09 (2015) 057 [arXiv: 1505.00226] [INSPIRE].

[24] CMS collaboration, Measurements of properties of the Higgs boson decaying into the four-lepton final state in pp collisions at $\sqrt{s}=13 \mathrm{TeV}$, JHEP 11 (2017) 047 [arXiv: 1706. 09936] [INSPIRE].

[25] CMS collaboration, Higgs to WW measurements with $15.2 \mathrm{fb}^{-1}$ of 13 TeV proton-proton collisions, CMS-PAS-HIG-16-021 (2016).

[26] CMS collaboration, Observation of the Higgs boson decay to a pair of $\tau$ leptons with the CMS detector, Phys. Lett. B 779 (2018) 283 [arXiv:1708.00373] [INSPIRE].

[27] ATLAS collaboration, Measurement of the Higgs boson coupling properties in the $H \rightarrow Z Z^{*} \rightarrow 4 \ell$ decay channel at $\sqrt{s}=13 \mathrm{TeV}$ with the ATLAS detector, ATLAS-CONF-2017-043 (2017).

[28] D. Foreman-Mackey, D.W. Hogg, D. Lang and J. Goodman, emcee: The MCMC Hammer, Publ. Astron. Soc. Pac. 125 (2013) 306 [arXiv:1202.3665] [INSPIRE].

[29] J. de Blas et al., Electroweak precision constraints at present and future colliders, PoS (ICHEP2016) 690 (2017) [arXiv: 1611.05354] [INSPIRE].

[30] G.C. Branco, P.M. Ferreira, L. Lavoura, M.N. Rebelo, M. Sher and J.P. Silva, Theory and phenomenology of two-Higgs-doublet models, Phys. Rept. 516 (2012) 1 [arXiv:1106.0034] [INSPIRE].

[31] N. Chakrabarty, U.K. Dey and B. Mukhopadhyaya, High-scale validity of a two-Higgs doublet scenario: a study including LHC data, JHEP 12 (2014) 166 [arXiv:1407.2145] [INSPIRE].

[32] A. Melfo, M. Nemevšek, F. Nesti, G. Senjanović and Y. Zhang, Type II Seesaw at LHC: The Roadmap, Phys. Rev. D 85 (2012) 055018 [arXiv:1108.4416] [InSPIRE].

[33] C.-S. Chen, C.-Q. Geng, D. Huang and L.-H. Tsai, New Scalar Contributions to $h \rightarrow Z \gamma$, Phys. Rev. D 87 (2013) 075019 [arXiv: 1301.4694] [INSPIRE].

[34] C.-S. Chen, C.-Q. Geng, D. Huang and L.-H. Tsai, $h \rightarrow Z \gamma$ in Type-II seesaw neutrino model, Phys. Lett. B 723 (2013) 156 [arXiv:1302.0502] [INSPIRE].

[35] A.G. Akeroyd and S. Moretti, Enhancement of $H \rightarrow \gamma \gamma$ from doubly charged scalars in the Higgs Triplet Model, Phys. Rev. D 86 (2012) 035015 [arXiv:1206.0535] [InSPIRE].

[36] P.S. Bhupal Dev, D.K. Ghosh, N. Okada and I. Saha, $125 \mathrm{GeV}$ Higgs Boson and the Type-II Seesaw Model, JHEP 03 (2013) 150 [Erratum ibid. 05 (2013) 049] [arXiv:1301.3453] [INSPIRE].

[37] A. Chaudhuri, W. Grimus and B. Mukhopadhyaya, Doubly charged scalar decays in a type-II seesaw scenario with two Higgs triplets, JHEP 02 (2014) 060 [arXiv:1305.5761] [INSPIRE].

[38] U. Haisch, $\bar{B} \rightarrow X_{s} \gamma:$ Standard Model and Beyond, arXiv:0805.2141 [INSPIRE].

[39] F. Mahmoudi and O. Stal, Flavor constraints on the two-Higgs-doublet model with general Yukawa couplings, Phys. Rev. D 81 (2010) 035016 [arXiv:0907.1791] [InSPIRE].

[40] R.S. Gupta and J.D. Wells, Next Generation Higgs Bosons: Theory, Constraints and Discovery Prospects at the Large Hadron Collider, Phys. Rev. D 81 (2010) 055012 [arXiv: 0912.0267] [INSPIRE].

[41] M. Jung, A. Pich and P. Tuzon, Charged-Higgs phenomenology in the Aligned two-Higgs-doublet model, JHEP 11 (2010) 003 [arXiv:1006.0470] [INSPIRE]. 
[42] M. Misiak et al., Updated NNLO QCD predictions for the weak radiative B-meson decays, Phys. Rev. Lett. 114 (2015) 221801 [arXiv: 1503.01789] [INSPIRE].

[43] J. Bernon, J.F. Gunion, H.E. Haber, Y. Jiang and S. Kraml, Scrutinizing the alignment limit in two-Higgs-doublet models: $m_{h}=125 \mathrm{GeV}$, Phys. Rev. D 92 (2015) 075004 [arXiv: 1507.00933] [INSPIRE].

[44] F. del Aguila, J.A. Aguilar-Saavedra, J. de Blas and M. Pérez-Victoria, Electroweak constraints on see-saw messengers and their implications for LHC, in Proceedings, 43rd Rencontres de Moriond on Electroweak Interactions and Unified Theories: La Thuile, Italy, March 1-8, 2008, pp. 45-52, 2008, arXiv:0806.1023 [INSPIRE].

[45] A. Arhrib et al., Collider Signatures for Heavy Lepton Triplet in Type I+III Seesaw, Phys. Rev. D 82 (2010) 053004 [arXiv: 0904.2390] [INSPIRE].

[46] A.G. Akeroyd and M. Aoki, Single and pair production of doubly charged Higgs bosons at hadron colliders, Phys. Rev. D 72 (2005) 035011 [hep-ph/0506176] [INSPIRE].

[47] A.G. Akeroyd and C.-W. Chiang, Doubly charged Higgs bosons and three-lepton signatures in the Higgs Triplet Model, Phys. Rev. D 80 (2009) 113010 [arXiv: 0909.4419] [InSPIRE].

[48] A.G. Akeroyd and C.-W. Chiang, Phenomenology of Large Mixing for the CP-even Neutral Scalars of the Higgs Triplet Model, Phys. Rev. D 81 (2010) 115007 [arXiv:1003.3724] [INSPIRE].

[49] A.G. Akeroyd and H. Sugiyama, Production of doubly charged scalars from the decay of singly charged scalars in the Higgs Triplet Model, Phys. Rev. D 84 (2011) 035010 [arXiv:1105.2209] [INSPIRE].

[50] S. Chakrabarti, D. Choudhury, R.M. Godbole and B. Mukhopadhyaya, Observing doubly charged Higgs bosons in photon-photon collisions, Phys. Lett. B 434 (1998) 347 [hep-ph/9804297] [INSPIRE].

[51] K. Cheung and D.K. Ghosh, Triplet Higgs boson at hadron colliders, JHEP 11 (2002) 048 [hep-ph/0208254] [INSPIRE].

[52] C.-W. Chiang, T. Nomura and K. Tsumura, Search for doubly charged Higgs bosons using the same-sign diboson mode at the LHC, Phys. Rev. D 85 (2012) 095023 [arXiv:1202.2014] [INSPIRE].

[53] S. Kanemura, K. Yagyu and H. Yokoya, First constraint on the mass of doubly-charged Higgs bosons in the same-sign diboson decay scenario at the LHC, Phys. Lett. B 726 (2013) 316 [arXiv: 1305.2383] [INSPIRE].

[54] Z. Kang, J. Li, T. Li, Y. Liu and G.-Z. Ning, Light Doubly Charged Higgs Boson via the $W W^{*}$ Channel at LHC, Eur. Phys. J. C 75 (2015) 574 [arXiv:1404.5207] [INSPIRE].

[55] S. Kanemura, M. Kikuchi, K. Yagyu and H. Yokoya, Bounds on the mass of doubly-charged Higgs bosons in the same-sign diboson decay scenario, Phys. Rev. D 90 (2014) 115018 [arXiv: 1407.6547] [INSPIRE].

[56] S. Kanemura, M. Kikuchi, H. Yokoya and K. Yagyu, LHC Run-I constraint on the mass of doubly charged Higgs bosons in the same-sign diboson decay scenario, PTEP 2015 (2015) 051B02 [arXiv: 1412.7603] [INSPIRE].

[57] C.-H. Chen and T. Nomura, Search for $\delta^{ \pm \pm}$with new decay patterns at the LHC, Phys. Rev. D 91 (2015) 035023 [arXiv:1411.6412] [INSPIRE].

[58] Z.-L. Han, R. Ding and Y. Liao, LHC Phenomenology of Type II Seesaw: Nondegenerate Case, Phys. Rev. D 91 (2015) 093006 [arXiv:1502.05242] [INSPIRE]. 
[59] Z.-L. Han, R. Ding and Y. Liao, LHC phenomenology of the type-II seesaw mechanism: Observability of neutral scalars in the nondegenerate case, Phys. Rev. D 92 (2015) 033014 [arXiv: 1506.08996] [INSPIRE].

[60] M. Mitra, S. Niyogi and M. Spannowsky, Type-II Seesaw Model and Multilepton Signatures at Hadron Colliders, Phys. Rev. D 95 (2017) 035042 [arXiv:1611.09594] [InSPIRE].

[61] D.K. Ghosh, N. Ghosh, I. Saha and A. Shaw, Revisiting the high-scale validity of the type-II seesaw model with novel LHC signature, Phys. Rev. D 97 (2018) 115022 [arXiv:1711.06062] [INSPIRE].

[62] J.C. Pati and A. Salam, Lepton Number as the Fourth Color, Phys. Rev. D 10 (1974) 275 [Erratum ibid. D 11 (1975) 703] [INSPIRE].

[63] R.N. Mohapatra and J.C. Pati, Left-Right Gauge Symmetry and an Isoconjugate Model of CP-violation, Phys. Rev. D 11 (1975) 566 [InSPIRE].

[64] R.N. Mohapatra and J.C. Pati, A Natural Left-Right Symmetry, Phys. Rev. D 11 (1975) 2558 [INSPIRE].

[65] A. Chaudhuri and B. Mukhopadhyaya, CP-violating phase in a two Higgs triplet scenario: Some phenomenological implications, Phys. Rev. D 93 (2016) 093003 [arXiv:1602.07846] [INSPIRE].

[66] D. Kumar Ghosh, N. Ghosh and B. Mukhopadhyaya, Distinctive Collider Signals for a Two Higgs Triplet Model, arXiv: 1808.01775 [INSPIRE]. 